\title{
Stability analysis and finite volume element discretization for delay-driven spatio-temporal patterns in a predator-prey model
}

\author{
Raimund Bürger \\ $C I^{2} M A$ and Departamento de Ingeniería Matemática, Universidad de Concepción, Casilla 160-C, Concepción, \\ Chile. Email: rburger@ing-mat.udec.cl \\ Ricardo Ruiz-Baier \\ Mathematical Institute, Oxford University, A. Wiles Building, Radcliffe Observatory Quarter, Woodstock Road, \\ OX2 6GG Oxford, UK. Email: ruizbaier@maths.ox.ac.uk \\ Canrong Tian \\ Department of Basic Sciences, Yancheng Institute of Technology, Yancheng 224003, China. Email: \\ tiancanrong@163.com
}

\begin{abstract}
Time delay is an essential ingredient of spatio-temporal predator-prey models since the reproduction of the predator population after predating the prey will not be instantaneous, but is mediated by a constant time lag accounting for the gestation of predators. In this paper we study a predator-prey reaction-diffusion system with time delay, where a stability analysis involving Hopf bifurcations with respect to the delay parameter and simulations produced by a new numerical method reveal how this delay affects the formation of spatial patterns in the distribution of the species. In particular, it turns out that when the carrying capacity of the prey is large and whenever the delay exceeds a critical value, the reaction-diffusion system admits a limit cycle due to the Hopf bifurcation. This limit cycle induces the spatio-temporal pattern. The proposed discretization consists of a finite volume element (FVE) method combined with a Runge-Kutta scheme.
\end{abstract}

Keywords: Spatio-temporal patterns, Time delay, Limit cycle, Pattern selection, Finite volume element discretization

2000 MSC: 35B35, 35B40, 65M60, 92D40

\section{Introduction}

\subsection{Scope}

The effect of time delay is fundamental in continuous models of populations of single or multiple species whenever the growth rate of a population does not respond instantaneously to changes in population size. One of the first models with delay was proposed by Volterra [55], who took into account the delay in response of a population's death rate to changes in population density caused by an accumulation of pollutants in the past. Further causes of response delays include differences in resource consumption with respect to age structure, migration and diffusion of populations, gestation and maturation periods, delays in behavioural response to environmental changes, and dependence of a population on a food supply that requires time to recover from grazing [7. Within epidemic 
models, time delays describe the incubation periods of infectious diseases, the infection periods of infective members, and the periods of recovered individuals with immunity [57. More generally, the main expected consequence of including time delay is oscillatory solution behaviour [41].

In this work we are interested in criteria for the formation, and numerical methods for the efficient simulation, of spatio-temporal patterns described by a predator-prey model with time delay and diffusion. The model is given by the following initial-boundary value problem for a pair of reaction-diffusion equations, the second of them with a delay term:

$$
\begin{aligned}
& \partial_{t} u_{1}-d_{1} \Delta u_{1}=u_{1}\left(a_{1}-b_{11} u_{1}-b_{12} u_{2}\right), \quad(\boldsymbol{x}, t) \in \Omega \times \mathcal{T}, \\
& \partial_{t} u_{2}-d_{2} \Delta u_{2}=u_{2}\left(-a_{2}+b_{21}\left(u_{1}\right)_{\tau}-b_{22} u_{2}\right), \quad(\boldsymbol{x}, t) \in \Omega \times \mathcal{T}, \\
& \partial_{\boldsymbol{n}} u_{1}=\partial_{\boldsymbol{n}} u_{2}=0, \quad(\boldsymbol{x}, t) \in \Sigma_{\mathcal{T}}, \\
& u_{1}(\boldsymbol{x}, t)=\psi_{1}(\boldsymbol{x}, t), \quad u_{2}(\boldsymbol{x}, t)=\psi_{2}(\boldsymbol{x}, t), \quad(\boldsymbol{x}, t) \in \Omega_{\tau} .
\end{aligned}
$$

The model is posed on a finite time interval $\mathcal{T}:=(0, T)$ for a fixed final time $T>0$, and where $\Sigma_{\mathcal{T}}:=(\partial \Omega) \times \mathcal{T}, \Omega_{\tau}:=\Omega \times[-\tau, 0]$, and $\partial_{\boldsymbol{n}}$ denotes the directional derivative with respect to the outer normal vector $\boldsymbol{n}$ of the boundary $\partial \Omega$ of $\Omega$. Here $u_{1}=u_{1}(\boldsymbol{x}, t)$ and $u_{2}=u_{2}(\boldsymbol{x}, t)$ are the sought densities of the prey and the predator, respectively. The right-hand side of $(1.1 \mathrm{~b})$ includes the delay term $\left(u_{1}\right)_{\tau}:=u_{1}(\boldsymbol{x}, t-\tau)$, where the constant $\tau>0$ is the delay. The delay in $(1.1 \mathrm{~b})$ can be regarded as a gestation period (roughly speaking, abundance of prey at time $t$ will influence the grow of the predator population at time $t+\tau$ ) or reaction time of the predators [48. The homogeneous Neumann boundary condition $1.1 \mathrm{c}$ indicates zero population flux across $\partial \Omega$. Moreover, the parameters $a_{1}$ and $a_{2}$ are, respectively, the growth rate of the prey and the death rate of the predator. Both are assumed strictly positive for sake of the subsequent analysis. In addition $b_{i i}(i=1,2)$ are the rates of intra-specific competition (assumed nonzero), and $b_{12}$ and $b_{21}$ denote the rates of consumption by predator on prey and mass conversion from prey to predator, respectively. The ratios $a_{i} / b_{i i}(i=1,2)$ are environmental carrying capacities, and $d_{1}$ and $d_{2}$ are diffusion coefficients of each species.

The first purpose of this paper to study the spatio-temporal patterns produced by solutions of 1.1) and to examine the onset of oscillatory solution behaviour through a Hopf bifurcation with respect to the delay $\tau$ as a bifurcation parameter. The second purpose is to introduce a new numerical method for the solution of (1.1). Our objective here is to explore how delay determines the stability threshold of the steady state of 1.1a), 1.1b). The present analysis reveals that spatio-temporal patterns can be induced by a series of Hopf bifurcation critical points. Specifically, spatio-temporal patterns become possible for supercritical values of delay when the limit cycle appears due to the Hopf bifurcation. To the authors' knowledge, the formation of spatio-temporal patterns as a consquence of delay has not yet been reported in the literature related to spatial patterns. Nevertheless, there is a body of work in investigating pattern formation in reaction-diffusion systems due to the existence of a limit cycle; see, for instance, [36, 39.

\subsection{Related work}

Introductions to delay differential equations are given by Kuang [33] and Smith [52]; see also Chapter 8 of McKibben [38. For general introductions to bifurcation theory we mention [13] and [27, as well as [28] for Hopf bifurcations. In predator-prey systems, delay effects were first considered by Volterra [56]. He showed that under certain conditions, all solutions possess an oscillatory behaviour. In fact, there are many plausible ways to introduce delays into a predator-prey model, see [48] (and the references cited therein) for a survey in the non-spatial setting. For the delayed non-spatial predator-prey model, the asymptotic stability of the equilibrium and the periodicity of the solution were investigated (see [6, 16, 37, and the references therein). Analyses of non-spatial variants of (1.1) also include [19, 31, 49, 58, 62, and references to spatio-temporal pattern formation include, besides [36, 39] and the vast list of references in both works, [14] and [25]. Numerical methods tailored for these kinds of problems can be found in e.g. [2, 26]. We here decide to use stable Runge-Kutta (RK) schemes proposed by Koto [32] (see also [29, 30]). 
For predator-prey models with diffusion, the existence of traveling wave solutions was shown in [40] and [22] for discrete and continuous delays, respectively, although the models differ from (1.1). For (1.1) and related spatio-temporal models, Gourley et al. 24] presents a survey of mechanisms where diffusion and time delays may coexist in a system involving nonlocal terms, in such a way that the ability of individuals to be at different points in space, at past times, can be explained. On the other hand, Sen et al. [50] show that the time delay may induce spatial patterns in the reactiondiffusion system. A version of (1.1) with Beddington-Angelis functional response is studied in 61] along with numerical simulations in one space dimension. We also mention [54], where the formation of delay-induced Turing patterns is analyzed for a version of (1.1) with a different functional response (the bifurcation theory in that paper is less involved). Bifurcations akin to those studied herein are also examined by [47] for a fully discrete coupled map lattice predator-prey model.

We study the effects of the time delay by a finite volume element (FVE) approximation of (1.1). This method is a hybrid concept between finite elements and finite volume discretizations that features some desirable properties including the ability to deal with unstructured meshes on arbitrarily shaped domains, the conservativity of inter-element fluxes, and the feasibility of error estimates in $L^{2}$ and $H^{1}$ norms. FVE methods have historically been applied for flow equations [11, 12, 34, 46 and recently also for several applicative time-dependent convection-diffusion problems [8, 9, 17, 18, 35, 45]. Other numerical methods for similar problems include finite differences [20, 53, 59, finite volume methods [1, 5, 51, and finite elements [4, 21, 60].

\subsection{Outline of the paper}

In Section 2 we show that without delay (i.e., $\tau=0$ ), the problem 1.1 does not generate spatial patterns, while in the presence of delay $(\tau>0)$, the formation of spatial patterns is induced. To this end, after stating some preliminaries (Section 2.1), we first prove (in Section 2.2 by standard arguments that 1.1 admits a unique, positive and uniformly bounded solution for all times. In Section 2.3 we analyze the linear stability of (1.1). We show that when $\tau$ exceeds a certain critical value $\tau^{*}$, then the operator arising from linearization of (1.1) around the non-trivial equilibrium $\boldsymbol{u}^{*}$ admits sequences $\left\{\tau_{n}^{*}\right\}_{n \in \mathbb{N}_{0}}$ of purely complex eigenvalues, and that the solutions of the linearized version undergo a Hopf bifurcation at $\boldsymbol{u}=\boldsymbol{u}^{*}$ whenever $\tau=\tau_{n}^{*}$. Next, in Section 2.4 we employ the normal form method and the center manifold theory to analyze the direction of the Hopf bifurcation of solutions of (1.1) (using $\tau$ as bifurcation parameter) obtained in Section 2.3. The result is a predictive criterion that states whether the Hopf bifurcations are supercritical or subcritical, respectively, and the corresponding bifurcating periodic solutions on the center manifold are stable (unstable, respectively). In Section 3 we introduce the numerical method for the solution of (1.1), which is based on a FVE spatial discretization (introduced in Section 3.1) combined with a RungeKutta method for delay differential equations (Section 3.2). Numerical results are presented in Section 4. Example 1 (Section 4.2 refers to a simplified version of 1.1), for which an exact solution is available. The recorded error histories indicate that the method converges when discretization parameters are refined. Examples 2 and 3 (Sections 4.3 and 4.4) consider the full model (1.1) on a square. It is illustrated that spatial pattern formation and temporal oscillatory behaviour appear as predicted. Example 4 in Section 4.5 reports similar findings in a disk-shaped domain, while Example 5 in Section 4.6 alerts to the formation of structures similar to spiral waves in a rectangular domain. Finally, Section 5 collects some conclusions.

\section{Delay-driven spatial patterns}

\subsection{Preliminaries}


Notation 1. Let $0=\mu_{1}<\mu_{2}<\cdots \rightarrow \infty$ be the eigenvalues of $-\Delta$ on $\Omega$ under no-flux boundary conditions, and $\mathcal{E}\left(\mu_{i}\right)$ be the space of eigenfunctions corresponding to $\mu_{i}$. We define the following space decomposition:

(i) $\mathcal{X}_{i j}:=\left\{\boldsymbol{c} \cdot \phi_{i j}: \boldsymbol{c} \in \mathbb{R}^{2}\right\}$, where $\left\{\phi_{i j}\right\}$ is an orthonormal basis of $\mathcal{E}\left(\mu_{i}\right)$ for $j=1, \ldots, \operatorname{dim} \mathcal{E}\left(\mu_{i}\right)$,

(ii) $\mathcal{X}:=\left\{\boldsymbol{u}=\left(u_{1}, u_{2}\right)^{\mathrm{T}} \in\left[C^{1}(\bar{\Omega})\right]^{2}: \partial_{\boldsymbol{n}} u_{1}=\partial_{\boldsymbol{n}} u_{2}=0\right.$ on $\left.\partial \Omega\right\}$. Thus,

$$
\mathcal{X}=\bigoplus_{i=1}^{\infty} \mathcal{X}_{i}, \quad \text { where } \mathcal{X}_{i}=\bigoplus_{j=1}^{\operatorname{dim} \mathcal{E}\left(\mu_{i}\right)} \mathcal{X}_{i j}
$$

Furthermore, we will eventually employ the following result (see Theorem 2.1 in [44]).

Lemma 2.1. Let $\left(\tilde{c}_{1}, \tilde{c}_{2}\right)$ and $\left(\hat{c}_{1}, \hat{c}_{2}\right)$ be a pair of ordered upper and lower solutions of the system (1.1). Then, that system has a unique global solution $\left(u_{1}(\boldsymbol{x}, t), u_{2}(\boldsymbol{x}, t)\right)$ such that $\hat{c}_{i} \leq u_{i}(\boldsymbol{x}, t) \leq \tilde{c}_{i}$, $i=1,2$, for $(\boldsymbol{x}, t) \in \Omega \times[0, \infty)$.

We will also appeal to the following lemma (proven in Appendix A of [19]).

Lemma 2.2 (Butler's lemma). Let $\alpha+\beta<0$ and $\alpha \beta>\gamma$. Then the real parts of solutions of $\lambda^{2}-(\alpha+\beta) \lambda+\alpha \beta-\gamma \mathrm{e}^{-\tau \lambda}=0$ are negative for $\tau<\tau_{0}$, where $\tau_{0}>0$ is the smallest value for which this equation has a solution with real part zero.

\subsection{Existence of a solution}

In order to establish the global existence of a solution to (1.1), it suffices to show that any solution candidate $\left(u_{1}(\boldsymbol{x}, t), u_{2}(\boldsymbol{x}, t)\right)$ must be bounded for all $t>0$. To this end, we define the quantities $\hat{\psi}_{i}(\tau):=\sup _{(y, s) \in \Omega_{\tau}} \psi_{i}(y, s)$ for $i=1,2$.

Theorem 2.3. The initial-boundary value problem with delay (1.1) has a unique solution $\left(u_{1}, u_{2}\right)$ for $T=\infty$. Moreover, the components $u_{1}$ and $u_{2}$ satisfy the following respective bounds:

$$
\begin{gathered}
0<u_{1}(\boldsymbol{x}, t) \leq \mathcal{A}_{1}(\tau):=\max \left\{\frac{a_{1}}{b_{11}}, \hat{\psi}_{1}(\tau)\right\} \\
0<u_{2}(\boldsymbol{x}, t) \leq \mathcal{A}_{2}(\tau):=\max \left\{\frac{1}{b_{22}}\left[b_{21} \max \left\{\frac{a_{1}}{b_{11}}, \hat{\psi}_{1}(\tau)\right\}-a_{2}\right], \hat{\psi}_{2}(\tau)\right\} .
\end{gathered}
$$

Proof. A straightforward derivation of local existence and uniqueness of solutions of $(1.1)$ can be provided for small values of $T$. We first show the positivity of the local solution $\left(u_{1}(\boldsymbol{x}, t), u_{2}(\boldsymbol{x}, t)\right)$ for some $T$ (here and in what follows, we understand by positivity of the pair $\left(u_{1}, u_{2}\right)$ that $u_{1}>0$ and $\left.u_{2}>0\right)$. We now consider (1.1) for the time interval $\mathcal{T}=(0, \tau)$. Since the initial values are positive, the term $\left(u_{1}\right)_{\tau}$ is positive and bounded on $\Omega \times(0, \tau)$. A direct application of the standard maximum principle for parabolic equations implies that the local solution $\left(u_{1}(\boldsymbol{x}, t), u_{2}(\boldsymbol{x}, t)\right)$ of 1.1 ) for $\mathcal{T}=(0, \tau)$ is positive on $\Omega \times(0, \tau]$. Moreover, the bounds 2.2$)$ and (2.3) are valid for $0<t<\tau$. In a similar way, we now consider the system formed by $1.1 \mathrm{a}), 11.1 \mathrm{~b})$ and $(1.1 \mathrm{c})$ for $\mathcal{T}=(\tau, 2 \tau)$ along with the initial condition $u_{1}(\boldsymbol{x}, t)=\psi_{1}(\boldsymbol{x}, t)$ and $u_{2}(\boldsymbol{x}, t)=\psi_{2}(\boldsymbol{x}, t)$ for $(\boldsymbol{x}, t) \in \Omega \times[0, \tau]$. Then the solution $\left(u_{1}(\boldsymbol{x}, t), u_{2}(\boldsymbol{x}, t)\right)$ of this system is positive on $\Omega \times(\tau, 2 \tau]$. By the induction principle, $\left(u_{1}(\boldsymbol{x}, t), u_{2}(\boldsymbol{x}, t)\right)$ is positive and bounded for some $T$.

Now we deduce the global existence of the solution $\left(u_{1}(\boldsymbol{x}, t), u_{2}(\boldsymbol{x}, t)\right)$ by appealing to the method of upper and lower solutions stated in Lemma 2.1. It is easy to verify that the pairs $\left(\mathcal{A}_{1}(\tau), \mathcal{A}_{2}(\tau)\right)$ 
and $(0,0)$ are ordered upper and lower solutions of 1.1). Now the existence theorem of [44] implies that (1.1) admits a unique global solution $\left(u_{1}(\boldsymbol{x}, t), u_{2}(\boldsymbol{x}, t)\right)$.

We observe that if the initial values $\psi_{i}(\boldsymbol{x}, 0), i=1,2$, are nonnegative and none of the initial values is identically zero, then the corresponding solution of 11.1$)\left(u_{1}(\boldsymbol{x}, t), u_{2}(\boldsymbol{x}, t)\right)$ is strictly positive on $\Omega \times \mathcal{T}$.

\subsection{Linear stability analysis}

The system 1.1a), 1.1b has a nontrivial equilibrium

$$
\boldsymbol{u}^{*}=\left(u_{1}^{*}, u_{2}^{*}\right)=\left(\frac{a_{1} b_{22}+a_{2} b_{12}}{b_{11} b_{22}+b_{12} b_{21}}, \frac{a_{1} b_{21}-a_{2} b_{11}}{b_{11} b_{22}+b_{12} b_{21}}\right),
$$

which is feasible (i.e., $u_{1}^{*}>0$ and $u_{2}^{*}>0$ ) if we assume that the parameters of the kinetics in (1.1) satisfy

$$
a_{1} / b_{11}>a_{2} / b_{21}
$$

We now set $w_{1}:=u_{1}-u_{1}^{*}, w_{2}:=u_{2}-u_{2}^{*}$ and substitute $u_{1}=w_{1}+u_{1}^{*}$ and $u_{2}=w_{2}+u_{2}^{*}$ into (1.1). This yields the following system for $w_{1}$ and $w_{2}$, which is equivalent to (1.1):

$$
\begin{aligned}
& \partial_{t} w_{1}-d_{1} \Delta w_{1}=\left(u_{1}^{*}+w_{1}\right)\left(-b_{11} w_{1}-b_{12} w_{2}\right), \quad(\boldsymbol{x}, t) \in \Omega_{T}, \\
& \partial_{t} w_{2}-d_{2} \Delta w_{2}=\left(u_{2}^{*}+w_{2}\right)\left(b_{21} w_{1}(\boldsymbol{x}, t-\tau)-b_{22} w_{2}\right), \quad(\boldsymbol{x}, t) \in \Omega_{T}, \\
& \partial_{\boldsymbol{n}} w_{1}=\partial_{\boldsymbol{n}} w_{2}=0, \quad(\boldsymbol{x}, t) \in \Sigma_{T}, \\
& w_{1}(\boldsymbol{x}, t)=\psi_{1}(\boldsymbol{x}, t)-u_{1}^{*}, \quad w_{2}(\boldsymbol{x}, t)=\psi_{2}(\boldsymbol{x}, t)-u_{2}^{*}, \quad(\boldsymbol{x}, t) \in \Omega_{\tau} .
\end{aligned}
$$

We retain only the linear terms in $w_{1}$ and $w_{2}$ to obtain the following linearization of 1.1 around $\boldsymbol{u}^{*}$ :

$$
\begin{aligned}
& \partial_{t} w_{1}-d_{1} \Delta w_{1}=-b_{11} u_{1}^{*} w_{1}-b_{12} u_{1}^{*} w_{2}, \quad(\boldsymbol{x}, t) \in \Omega_{T}, \\
& \partial_{t} w_{2}-d_{2} \Delta w_{2}=b_{21} u_{2}^{*} w_{1}(\boldsymbol{x}, t-\tau)-b_{22} u_{2}^{*} w_{2}, \quad(\boldsymbol{x}, t) \in \Omega_{T}, \\
& \partial_{\boldsymbol{n}} w_{1}=\partial_{\boldsymbol{n}} w_{2}=0, \quad(\boldsymbol{x}, t) \in \Sigma_{T}, \\
& w_{1}(\boldsymbol{x}, t)=\psi_{1}(\boldsymbol{x}, t)-u_{1}^{*}, \quad w_{2}(\boldsymbol{x}, t)=\psi_{2}(\boldsymbol{x}, t)-u_{2}^{*}, \quad(\boldsymbol{x}, t) \in \Omega_{\tau} .
\end{aligned}
$$

By applying standard manipulations for delay differential equations (cf., e.g., [52]), we therefore may express the linearization of 1.1] around $\boldsymbol{u}^{*}$ as $\partial_{t} \boldsymbol{u}=\left(\boldsymbol{D} \Delta+\boldsymbol{J}^{*}\right) \boldsymbol{w}$, where $\boldsymbol{D}=\operatorname{diag}\left(d_{1}, d_{2}\right)$, $\boldsymbol{w}=\left(w_{1}, w_{2}\right)^{\mathrm{T}}$, and

$$
\boldsymbol{J}^{*}=\left[\begin{array}{cc}
-b_{11} u_{1}^{*} & -b_{12} u_{1}^{*} \\
b_{21} u_{2}^{*} \mathrm{e}^{-\lambda \tau} & -b_{22} u_{2}^{*}
\end{array}\right] .
$$

Notation 1 implies that $\mathcal{X}_{i}$ is invariant under the operator $\boldsymbol{D} \Delta+\boldsymbol{J}^{*}$, and $\lambda$ is an eigenvalue of this operator on $\mathcal{X}_{i}$ if and only if it is also an eigenvalue of the matrix $-\mu_{i} \boldsymbol{D}+\boldsymbol{J}^{*}$. Let us now fix the index $i$ and notice that the characteristic equation of $-\mu_{i} \boldsymbol{D}+\boldsymbol{J}^{*}$ is

$$
\Delta(\lambda, \tau):=\operatorname{det}\left(-\mu_{i} \boldsymbol{D}+\boldsymbol{J}^{*}-\lambda\right)=0 .
$$

A direct calculation shows that

$$
\Delta(\lambda, \tau)=\lambda^{2}-R_{i} \lambda+Q \mathrm{e}^{-\lambda \tau},
$$

where for sake of brevity, we define

$$
R_{i}:=b_{11} u_{1}^{*}+b_{22} u_{2}^{*}+d_{1} \mu_{i}+d_{2} \mu_{i}>0, \quad Q:=b_{12} b_{21} u_{1}^{*} u_{2}^{*} .
$$

Then we have the following result on the existence of delay-driven spatial patterns. 
Theorem 2.4. If the parameters in the kinetics of (1.1) satisfy assumption (2.4), then the system (1.1) admits spatio-temporal patterns due to the presence of the delay. More precisely, the following holds:

(i) If the delay is absent, that is $\tau=0$, then the positive equilibrium $\boldsymbol{u}^{*}$ of 1.1 is locally asymptotically stable.

(ii) If the delay is present, that is $\tau \neq 0$, assume that $\omega^{*}<Q^{1 / 2}$, where

$$
\omega^{*}:=\frac{1}{\sqrt{2}}\left(\left[R_{i}^{4}+4 Q^{2}\right]^{1 / 2}-R_{i}^{2}\right)^{1 / 2},
$$

for $R_{i}$ and $Q$ as defined in 2.8). Then there exists a critical point

$$
\tau^{*}=\frac{1}{\omega^{*}} \arccos \frac{\omega^{* 2}}{b_{12} b_{21} u_{1}^{*} u_{2}^{*}}=\frac{1}{\omega^{*}} \arccos \frac{\omega^{* 2}}{Q},
$$

such that the positive equilibrium $\boldsymbol{u}^{*}$ is locally asymptotically stable for $\tau \in\left[0, \tau^{*}\right]$ and unstable for $\tau \in\left(\tau^{*}, \infty\right)$.

Proof. (i) We first show that $\tau=0$ does not generate spatial patterns. From the above argument, it is sufficient to show that all roots of $\Delta(\lambda, 0)$ have negative real parts. It follows from 2.7) that $\Delta(\lambda, 0)=\lambda^{2}-R_{i} \lambda+Q$. Therefore, by the Descartes rule of sign, the quadratic equation $\Delta(\lambda, 0)=0$ always has two negative roots.

(ii) In light of the theory developed in [23, Chapter 3], in order to prove the instability of the uniform equilibrium it is sufficient to show that there exist a purely imaginary number $\omega$ i, where $\omega \in \mathbb{R}$ and $\mathrm{i}=\sqrt{-1}$ is the imaginary unit, and a real number $\tau>0$ such that $\Delta(\omega \mathrm{i}, \tau)=0$. If $\omega \mathrm{i}$ is a root of 2.6 , then $\omega$ must satisfy the pair of equations

$$
-\omega^{2}-Q \cos \omega \tau=0, \quad \omega R_{i}-Q \sin \omega \tau=0,
$$

which leads to the quadratic equation (with respect to $\omega^{2}$ )

$$
\omega^{4}+R_{i}^{2} \omega^{2}-Q^{2}=0 .
$$

Again by the Descartes rule of sign, we have that this equation always has a unique positive real root $\omega=\omega^{*}$ given by $(2.9)$, and $\Delta(\omega \mathrm{i}, \tau)=0$ has a pair of simple purely imaginary roots $\pm \omega^{*} \mathrm{i}$ whenever

$$
\tau_{n}^{*}=\frac{1}{\omega^{*}} \arccos \frac{\omega^{* 2}}{Q}+\frac{2 n \pi}{\omega^{*}}, \quad n=0,1, \ldots
$$

If we now set $\tau^{*}:=\tau_{0}^{*}$, then by Lemma $2.2, \boldsymbol{u}^{*}$ is stable for $\tau<\tau^{*}$. On the other hand, if $\tau \geq \tau^{*}$, then (2.7) has a unique root on the imaginary axis. By the eigenvalue theory of [49], the sum of orders of the zeros of (2.7) for $\tau>\tau^{*}$ is equal to that of $\tau=\tau^{*}$. Then (2.7) for $\tau \in\left[\tau^{*}, \infty\right)$ has roots with positive real parts, which implies that $\boldsymbol{u}^{*}$ is locally asymptotically unstable for $\tau \geq \tau^{*}$.

Theorem 2.5. Under assumption (2.4), all solutions of problem (1.1) undergo a Hopf bifurcation at $\boldsymbol{u}^{*}$ when $\tau=\tau_{n}^{*}$ for $n \in \mathbb{N}_{0}$.

Proof. We have already shown that $\Delta(\omega \mathrm{i}, \tau)=0$ has a pair of simple purely imaginary roots $\pm \omega^{*} \mathrm{i}$ at $\tau_{n}^{*}$. Thus, to prove that a Hopf bifurcation occurs at $\tau=\tau_{n}^{*}$ for $n=0,1, \ldots$, we must still prove the following transversality property:

$$
\left.\frac{\mathrm{d}}{\mathrm{d} \tau}(\operatorname{Re}(\lambda(\tau)))\right|_{\tau=\tau_{n}^{*}}>0, \quad n=0,1, \ldots
$$


To this end, we substitute $\lambda=\sigma+\omega \mathrm{i}, \sigma, \omega \in \mathbb{R}$ into 2.6 to obtain

$$
\sigma^{2}-\omega^{2}-R_{i} \sigma+\mathrm{e}^{-\sigma \tau} Q \cos \omega \tau=0, \quad 2 \sigma \omega-R_{i} \omega-\mathrm{e}^{-\sigma \tau} Q \sin \omega \tau=0 .
$$

Differentiating these equations with respect to $\tau$ yields

$$
\begin{gathered}
\left(2 \sigma-R_{i}-\tau \mathrm{e}^{-\sigma \tau} Q \cos \omega \tau\right) \frac{\mathrm{d} \sigma}{\mathrm{d} \tau}-2 \omega \frac{\mathrm{d} \omega}{\mathrm{d} \tau}-\mathrm{e}^{-\sigma \tau} Q(\omega \sin \omega \tau+\sigma \cos \omega \tau)=0, \\
\left(2 \omega+\tau \mathrm{e}^{-\sigma \tau} Q \sin \omega \tau\right) \frac{\mathrm{d} \sigma}{\mathrm{d} \tau}+\left(2 \sigma-R_{i}\right) \frac{\mathrm{d} \omega}{\mathrm{d} \tau}+\mathrm{e}^{-\sigma \tau} Q(\sigma \sin \omega \tau-\omega \cos \omega \tau)=0 .
\end{gathered}
$$

Substituting $\sigma=0$ into the above equations, we obtain the system of linear equations

$$
\begin{gathered}
\left(-R_{i}-\tau Q \cos \omega \tau\right) \frac{\mathrm{d} \sigma}{\mathrm{d} \tau}-2 \omega \frac{\mathrm{d} \omega}{\mathrm{d} \tau}-Q \omega \sin \omega \tau=0 \\
(2 \omega+\tau Q \sin \omega \tau) \frac{\mathrm{d} \sigma}{\mathrm{d} \tau}-R_{i} \frac{\mathrm{d} \omega}{\mathrm{d} \tau}-Q \omega \cos \omega \tau=0
\end{gathered}
$$

whose solution gives

$$
\frac{\mathrm{d} \sigma}{\mathrm{d} \tau}=A^{-1}\left|\begin{array}{ll}
Q \omega \sin \omega \tau & -2 \omega \\
Q \omega \cos \omega \tau & -R_{i}
\end{array}\right|,
$$

where $A$ is the determinant. Substituting $\tau=\tau_{n}^{*}, \omega=\omega^{*}$ and 2.10 into the expression above, we obtain $A=R_{i}^{2}+4 \omega^{* 2}+\tau^{*} \omega^{* 2} R_{i}>0$, and thus

$$
\left.\frac{\mathrm{d} \sigma}{\mathrm{d} \tau}\right|_{\tau=\tau_{n}^{*}, \omega=\omega^{*}}=\frac{\left(Q \sin \omega^{*} \tau_{n}^{*}\right)^{2}+2 \omega^{* 4}}{A}>0,
$$

which proves 2.12.

\subsection{The direction and stability of the Hopf bifurcation}

As in Section 2.4 let us set $w_{1}:=u_{1}-u_{1}^{*}, w_{2}:=u_{2}-u_{2}^{*}$, and also $\gamma:=\tau-\tau_{n}^{*}$, so that $\gamma=0$ is the Hopf bifurcation value of system (1.1). Let us recall the Banach space decomposition (2.1) in Notation 1, and let us apply a rescaling of time $t \rightarrow t / \tau$, in order to normalize the delay. Then the PDEs 2.5a and $2.5 \mathrm{~b}$ can be written as follows:

$$
\begin{aligned}
& \partial_{t} w_{1}=\left(\tau_{n}^{*}+\gamma\right)\left(d_{1} \Delta w_{1}-b_{11} u_{1}^{*} w_{1}-b_{12} u_{1}^{*} w_{2}-b_{11} w_{1}^{2}-b_{12} w_{1} w_{2}\right), \\
& \partial_{t} w_{2}=\left(\tau_{n}^{*}+\gamma\right)\left(d_{2} \Delta w_{2}+b_{21} u_{2}^{*} w_{1}(\boldsymbol{x}, t-1)-b_{22} u_{2}^{*} w_{2}+b_{21} w_{1}(\boldsymbol{x}, t-1) w_{2}-b_{22} w_{2}^{2}\right) .
\end{aligned}
$$

We are now looking for solutions of (1.1), or equivalently, of (2.5) or of (2.13) (supplied with the appropriate initial and boundary conditions $2.5 \mathrm{c}$ and $2.5 \mathrm{~d}$ ) that are not repeated here) that are periodic in space in the sense that

$$
\Delta w_{l}+k^{2} w_{l}=0 \quad \text { on } \Omega \times \mathcal{T}, \quad \partial_{\boldsymbol{n}} w_{l}=0 \quad \text { on } \Sigma_{\mathcal{T}}, l=1,2,
$$

for a wavenumber $k$. According to Notation 1 , $k^{2}=\mu_{i}$ for a suitable index $i$, i.e., $\Delta w_{l}=-\mu_{i} w_{l}$ for a suitable index $i, l=1,2$. Inserting this into the right-hand sides of (2.13), we conclude that determining spatially periodic solutions of system $\sqrt{1.1}$ is equivalent to the solution of the following system:

$$
\begin{aligned}
& \partial_{t} w_{1}=\left(\tau_{n}^{*}+\gamma\right)\left(-d_{1} \mu_{i} w_{1}-b_{11} u_{1}^{*} w_{1}-b_{12} u_{1}^{*} w_{2}-b_{11} w_{1}^{2}-b_{12} w_{1} w_{2}\right), \\
& \partial_{t} w_{2}=\left(\tau_{n}^{*}+\gamma\right)\left(-d_{2} \mu_{i} w_{2}+b_{21} u_{2}^{*} w_{1}(\boldsymbol{x}, t-1)-b_{22} u_{2}^{*} w_{2}+b_{21} w_{1}(\boldsymbol{x}, t-1) w_{2}-b_{22} w_{2}^{2}\right) .
\end{aligned}
$$

Next, we use the notation of [28] and define the space $\mathcal{C}:=C\left([0,1], \mathbb{R}^{2}\right)$. Then the system $(2.14)$ is transformed into a functional differential equation as

$$
\dot{\boldsymbol{w}}(t)=L_{\gamma}\left(\boldsymbol{w}_{t}\right)+\boldsymbol{f}\left(\gamma, \boldsymbol{w}_{t}\right)
$$


where $\boldsymbol{w}(t)=\left(w_{1}(t), w_{2}(t)\right)^{\mathrm{T}} \in \mathbb{R}^{2},{ }^{\cdot} \equiv \mathrm{d} / \mathrm{d} t$, and the operators $L_{\gamma}: \mathcal{C} \rightarrow \mathbb{R}^{2}$ and $\boldsymbol{f}: \mathbb{R} \times \mathcal{C} \rightarrow \mathbb{R}^{2}$ are represented by the respective equations

$$
\begin{aligned}
L_{\gamma}(\phi)= & \left(\tau_{n}^{*}+\gamma\right)\left[\begin{array}{cc}
-d_{1} \mu_{i}-b_{11} u_{1}^{*} & -b_{12} u_{1}^{*} \\
0 & -d_{2} \mu_{i}-b_{22} u_{2}^{*}
\end{array}\right]\left(\begin{array}{l}
\phi_{1}(0) \\
\phi_{2}(0)
\end{array}\right) \\
& +\left(\tau_{n}^{*}+\gamma\right)\left[\begin{array}{cc}
0 & 0 \\
b_{21} u_{2}^{*} & 0
\end{array}\right]\left(\begin{array}{l}
\phi_{1}(-1) \\
\phi_{2}(-1)
\end{array}\right) \\
\boldsymbol{f}(\gamma, \phi)= & \left(\tau_{n}^{*}+\gamma\right)\left(\begin{array}{l}
-b_{11} \phi_{1}^{2}(0)-b_{12} \phi_{1}(0) \phi_{2}(0) \\
b_{21} \phi_{1}(-1) \phi_{2}(0)-b_{22} \phi_{2}^{2}(0)
\end{array}\right),
\end{aligned}
$$

where $\phi(\theta)=\left(\phi_{1}(\theta), \phi_{2}(\theta)\right)^{\mathrm{T}}$ for $\left(\phi_{1}, \phi_{2}\right)^{\mathrm{T}} \in \mathcal{C}$. By the Riesz representation theorem, there exists a $2 \times 2$ matrix, denoted $\eta(\theta, \gamma)$, whose entries are functions of bounded variation, such that

$$
L_{\gamma} \phi=\int_{-1}^{0}[\mathrm{~d} \eta(\theta, \gamma)] \phi(\theta) \quad \text { for } \phi \in \mathcal{C} .
$$

As a matter of fact, we can choose

$$
\eta(\theta, \gamma)=\left(\tau_{n}^{*}+\gamma\right)\left[\begin{array}{cc}
-d_{1} \mu_{i}-b_{11} u_{1}^{*} & -b_{12} u_{1}^{*} \\
0 & -d_{2} \mu_{i}-b_{22} u_{2}^{*}
\end{array}\right] \delta(\theta)+\left(\tau_{n}^{*}+\gamma\right)\left[\begin{array}{cc}
0 & 0 \\
b_{21} u_{2}^{*} & 0
\end{array}\right] \delta(\theta+1),
$$

where $\delta$ is a Dirac delta function. For $\phi \in C^{1}\left([-1,0], \mathbb{R}^{2}\right)$ we define

$$
A(\gamma) \phi:=\left\{\begin{array}{ll}
\frac{\mathrm{d} \phi(\theta)}{\mathrm{d} \theta} & \text { if } \theta \in[-1,0), \\
\int_{-1}^{0}[\mathrm{~d} \eta(s, \gamma)] \phi(s) & \text { if } \theta=0,
\end{array} \quad R(\gamma) \phi:= \begin{cases}0 & \text { if } \theta \in[-1,0), \\
f(\gamma, \phi) & \text { if } \theta=0 .\end{cases}\right.
$$

Thus, the system 2.15 is equivalent to

$$
\dot{\boldsymbol{w}}_{t}=A(\gamma)\left(\boldsymbol{w}_{t}\right)+R(\gamma)\left(\boldsymbol{w}_{t}\right),
$$

where $\boldsymbol{w}_{t}(\theta):=\boldsymbol{w}(t+\theta)$ for $\theta \in[-1,0]$. Now, for $\boldsymbol{\psi} \in C^{1}\left([0,1],\left(\mathbb{R}^{2}\right)^{*}\right)$ we define

$$
A^{*} \boldsymbol{\psi}(s):= \begin{cases}-\frac{\mathrm{d} \boldsymbol{\psi}(s)}{\mathrm{d} s} & \text { if } s \in[-1,0), \\ \int_{-1}^{0} \psi(-t) \mathrm{d} \eta^{\mathrm{T}}(t, 0) & \text { if } s=0\end{cases}
$$

and an inner product $\langle\cdot, \cdot\rangle$ by

$$
\langle\boldsymbol{\psi}(s), \boldsymbol{\phi}(\theta)\rangle=\overline{\boldsymbol{\psi}}(0) \boldsymbol{\phi}(0)-\int_{-1}^{0} \int_{0}^{\theta} \overline{\boldsymbol{\psi}}(\xi-\theta) \mathrm{d} \eta(\theta) \phi(\xi) \mathrm{d} \xi,
$$

where $\eta(\theta)=\eta(\theta, 0)$. Then $A(0)$ and $A^{*}$ are adjoint operators. From the discussion in Theorem 2.4. we know that $\pm \omega^{*} \tau_{n}^{*} \mathrm{i}$ are eigenvalues of $A(0)$ and, therefore, also of $A^{*}$. We now suppose that $\boldsymbol{q}(\theta)=\left(q_{1}, q_{2}\right)^{\mathrm{T}} \mathrm{e}^{\mathrm{i} \omega^{*} \tau_{n}^{*}}$ is the eigenvector of $A(0)$ corresponding to the eigenvalue $\omega^{*} \tau_{n}^{*} \mathrm{i}$. Thus, $A(0) \boldsymbol{q}(\theta)=\omega^{*} \tau_{n}^{*} \mathrm{i} \boldsymbol{q}(\theta)$, and from the definition of $A(0)$ we have

$$
\left[\begin{array}{cc}
-d_{1} \mu_{i}-b_{11} u_{1}^{*}-\mathrm{i} \omega^{*} & -b_{12} u_{1}^{*} \\
b_{21} u_{2}^{*} \mathrm{e}^{-\mathrm{i} \omega^{*} \tau_{n}^{*}} & -d_{2} \mu_{i}-b_{22} u_{2}^{*}-\mathrm{i} \omega^{*}
\end{array}\right]\left(\begin{array}{l}
q_{1} \\
q_{2}
\end{array}\right)=\mathbf{0},
$$

which implies that

$$
\boldsymbol{q}(\theta)=\left(1,-\frac{d_{1} \mu_{i}+b_{11} u_{1}^{*}+\mathrm{i} \omega^{*}}{b_{12} u_{1}^{*}}\right)^{\mathrm{T}} \mathrm{e}^{\mathrm{i} \omega^{*} \tau_{n}^{*} \theta}
$$


Similarly, let $\boldsymbol{q}^{*}(s)=M\left(q_{1}^{*}, q_{2}^{*}\right) \mathrm{e}^{\mathrm{i} \omega^{*} \tau_{n}^{*} s}$ be the eigenvector of $A^{*}$ corresponding to $-\mathrm{i} \omega^{*} \tau_{n}^{*}$, where the factor $M$ is to be determined later. Then by $A^{*} \boldsymbol{q}^{*}(s)=-\mathrm{i} \omega^{*} \tau_{n}^{*} \boldsymbol{q}^{*}(s)$ and the definition of $A^{*}$, we have

$$
\left[\begin{array}{cc}
-d_{1} \mu_{i}-b_{11} u_{1}^{*}+\mathrm{i} \omega^{*} & b_{21} u_{2}^{*} \mathrm{e}^{\mathrm{i} \omega^{*} \tau_{n}^{*}} \\
-b_{12} u_{1}^{*} & -d_{2} \mu_{i}-b_{22} u_{2}^{*}+\mathrm{i} \omega^{*}
\end{array}\right]\left(\begin{array}{l}
q_{1}^{*} \\
q_{2}^{*}
\end{array}\right)=\mathbf{0},
$$

which gives

$$
\boldsymbol{q}^{*}(s)=M\left(\frac{-d_{2} \mu_{i}-b_{22} u_{2}^{*}+\mathrm{i} \omega^{*}}{b_{12} u_{1}^{*}}, 1\right) \mathrm{e}^{\mathrm{i} \omega^{*} \tau_{n}^{*} s} .
$$

To ensure that $\left\langle\boldsymbol{q}^{*}(s), \boldsymbol{q}(\theta)\right\rangle=1$ it suffices with specifying $M$. From 2.18, we have

$$
\begin{aligned}
\left\langle\boldsymbol{q}^{*}(s), \boldsymbol{q}(\theta)\right\rangle & =\overline{\boldsymbol{q}}^{*}(0) \boldsymbol{q}(0)-\int_{-1}^{0} \int_{0}^{\theta} \overline{\boldsymbol{q}}^{*}(\xi-\theta) \mathrm{d} \eta(\theta) \boldsymbol{q}(\xi) \mathrm{d} \xi \\
& =\bar{M}\left(\bar{q}_{1}^{*}, \bar{q}_{2}^{*}\right)\left(\begin{array}{c}
q_{1} \\
q_{2}
\end{array}\right)-\int_{-1}^{0} \int_{0}^{\theta} \bar{M}\left(\bar{q}_{1}^{*}, \bar{q}_{2}^{*}\right) \mathrm{e}^{-\mathrm{i} \omega^{*} \tau_{n}^{*}(\xi-\theta)} \mathrm{d} \eta(\theta)\left(\begin{array}{c}
q_{1} \\
q_{2}
\end{array}\right) \mathrm{e}^{\mathrm{i} \omega^{*} \tau_{n}^{*} \xi} \mathrm{d} \xi \\
& =\bar{M} \bar{q}_{1}^{*}\left(q_{1}+\tau_{n}^{*} \mathrm{e}^{-\mathrm{i} \omega^{*} \tau_{n}^{*}}\left(0 q_{1}+0 q_{2}\right)\right)+\bar{M} \bar{q}_{2}^{*}\left(q_{2}+\tau_{n}^{*} \mathrm{e}^{-\mathrm{i} \omega^{*} \tau_{n}^{*}}\left(b_{21} u_{2}^{*} q_{1}+0 q_{2}\right)\right) \\
& =\bar{M}\left(\bar{q}_{1}^{*} q_{1}+\bar{q}_{2}^{*} q_{2}+\bar{q}_{2}^{*} q_{1} \tau_{n}^{*} b_{21} u_{2}^{*} \mathrm{e}^{-\mathrm{i} \omega^{*} \tau_{n}^{*}}\right) .
\end{aligned}
$$

Since $q_{1}=1$ and $q_{2}^{*}=1$, we can choose $M$ as

$$
\bar{M}=\left(\bar{q}_{1}^{*}+q_{2}+\tau_{n}^{*} b_{21} u_{2}^{*} \mathrm{e}^{-\mathrm{i} \omega^{*} \tau_{n}^{*}}\right)^{-1},
$$

where $\bar{M}$ is the conjugate imaginary of $M$. Next we will compute the coordinate to describe the center manifold $C_{0}$ at $\gamma=0$. Let $\boldsymbol{w}_{t}$ be the solution of (2.17) when $\gamma=0$. Define

$$
z(t):=\left\langle\boldsymbol{q}^{*}, \boldsymbol{w}_{t}\right\rangle, \quad \boldsymbol{W}(t, \theta):=\boldsymbol{w}_{t}(\theta)-2 \operatorname{Re}\{z(t) \boldsymbol{q}(\theta)\} .
$$

On the center manifold $C_{0}$, we have $\boldsymbol{W}(t, \theta)=\boldsymbol{W}(z(t), \bar{z}(t), \theta)$, where

$$
\boldsymbol{W}(z(t), \bar{z}(t), \theta)=\boldsymbol{W}_{20}(\theta) \frac{z^{2}}{2}+\boldsymbol{W}_{11}(\theta) z \bar{z}+\boldsymbol{W}_{02}(\theta) \frac{\bar{z}^{2}}{2}+\cdots,
$$

where $z$ and $\bar{z}$ are local coordinates for the center manifold $C_{0}$ in the direction of $\boldsymbol{q}^{*}$ and $\overline{\boldsymbol{q}}^{*}$. Recall that we only need to focus on real solutions, and note that $\boldsymbol{W}$ is real whenever $\boldsymbol{w}_{t}$ is real. For $\boldsymbol{w}_{t} \in C_{0}$ of 2.17), thanks to 2.19) and the fact that $\gamma=0$, we readily have

$$
\begin{aligned}
\dot{z}(t)=\left\langle\boldsymbol{q}^{*}, \dot{\boldsymbol{w}}_{t}\right\rangle & =\mathrm{i} \omega^{*} \tau_{n}^{*} z+\overline{\boldsymbol{q}}^{*}(0) \boldsymbol{f}(0, \boldsymbol{W}(z, \bar{z}, \theta)+2 \operatorname{Re}\{z \boldsymbol{q}(\theta)\}) \\
& \triangleq \mathrm{i} \omega^{*} \tau_{n}^{*} z+\overline{\boldsymbol{q}}^{*}(0) \boldsymbol{f}_{0}(z, \bar{z}),
\end{aligned}
$$

and we can rewrite (2.21) as $\dot{z}(t)=\mathrm{i} \omega^{*} \tau_{n}^{*} z+g(z, \bar{z})$, where

$$
g(z, \bar{z}):=\overline{\boldsymbol{q}}^{*}(0) \boldsymbol{f}_{0}(z, \bar{z})=g_{20} \frac{z^{2}}{2}+g_{11} z \bar{z}+g_{02} \frac{\bar{z}^{2}}{2}+g_{21} \frac{\bar{z}^{2} \bar{z}}{2}+\cdots .
$$

From 2.19 and 2.20 , we obtain that

$$
\begin{aligned}
\boldsymbol{w}_{t}(\theta)= & \boldsymbol{W}(t, \theta)+2 \operatorname{Re}\{z(t) \boldsymbol{q}(\theta)\} \\
= & \boldsymbol{W}_{20}(\theta) \frac{z^{2}}{2}+\boldsymbol{W}_{11}(\theta) z \bar{z}+\boldsymbol{W}_{02}(\theta) \frac{\bar{z}^{2}}{2}+z \boldsymbol{q}+\bar{z} \overline{\boldsymbol{q}}+\cdots \\
= & \boldsymbol{W}_{20}(\theta) \frac{z^{2}}{2}+\boldsymbol{W}_{11}(\theta) z \bar{z}+\boldsymbol{W}_{02}(\theta) \frac{\bar{z}^{2}}{2} \\
& +\mathrm{e}^{\mathrm{i} \omega^{*} \tau_{n}^{*} \theta} z\left(\begin{array}{c}
q_{1} \\
q_{2}
\end{array}\right)+\mathrm{e}^{-\mathrm{i} \omega^{*} \tau_{n}^{*} \theta} \bar{z}\left(\begin{array}{c}
\bar{q}_{1} \\
\bar{q}_{2}
\end{array}\right)+\cdots
\end{aligned}
$$


Substituting 2.16 and 2.23 into 2.22, we have

$$
\begin{aligned}
g(z, \bar{z}) & =\overline{\boldsymbol{q}}^{*}(0) \boldsymbol{f}_{0}(z, \bar{z})=\bar{M} \tau_{n}^{*}\left(\bar{q}_{1}^{*}, \bar{q}_{2}^{*}\right)\left(\begin{array}{l}
-b_{11} \phi_{1}^{2}(0)-b_{12} \phi_{1}(0) \phi_{2}(0) \\
b_{21} \phi_{1}(-1) \phi_{2}(0)-b_{22} \phi_{2}^{2}(0)
\end{array}\right) \\
& =p_{1} z^{2}+p_{2} z \bar{z}+p_{3} \bar{z}^{2}+p_{4} z^{2} \bar{z}+\text { h.o.t., }
\end{aligned}
$$

where h.o.t. stands for higher order terms, and

$$
\begin{aligned}
& p_{1}=\bar{M} \tau_{n}^{*}\left[\bar{q}_{1}^{*}\left(-b_{11}-b_{12} q_{2}\right)+\left(-b_{22} q_{2}^{2}+b_{21} q_{2} \mathrm{e}^{-\mathrm{i} \omega^{*} \tau_{n}^{*}}\right)\right], \\
& p_{2}=2 \bar{M} \tau_{n}^{*}\left[\bar{q}_{1}^{*}\left(-b_{11}-b_{12} \operatorname{Re}\left\{q_{2}\right\}\right)+\left(-b_{22}\left|q_{2}\right|^{2}+b_{21} \operatorname{Re}\left\{q_{2} \mathrm{e}^{\mathrm{i} \omega^{*} \tau_{n}^{*}}\right\}\right)\right] \text {, } \\
& p_{3}=\bar{M} \tau_{n}^{*}\left[\bar{q}_{1}^{*}\left(-b_{11}-b_{12} \bar{q}_{2}\right)+\left(-b_{22} \bar{q}_{2}^{2}+b_{21} \bar{q}_{2} \mathrm{e}^{\mathrm{i} \omega^{*} \tau_{n}^{*}}\right)\right] \text {, } \\
& p_{4}=\bar{M} \tau_{n}^{*} \bar{q}_{1}^{*}\left[-2 b_{11}\left(\frac{W_{20}^{(1)}(0)}{2}+W_{11}^{(1)}(0)\right)\right. \\
& \left.-b_{12}\left(\frac{W_{20}^{(1)}(0)}{2} \bar{q}_{2}+W_{11}^{(1)}(0) q_{2}+W_{11}^{(2)}(0)+\frac{W_{20}^{(2)}(0)}{2}\right)\right] \\
& +\bar{M} \tau_{n}^{*}\left[b_{21}\left(\frac{W_{20}^{(1)}(-1)}{2} \bar{q}_{2}+W_{11}^{(1)}(-1) q_{2}+W_{11}^{(2)}(0) \mathrm{e}^{-\mathrm{i} \omega \tau_{n}^{*}}+\frac{W_{20}^{(2)}(0)}{2} \mathrm{e}^{\mathrm{i} \omega \tau_{n}^{*}}\right)\right. \\
& \left.-2 b_{22}\left(\frac{W_{20}^{(2)}(0)}{2} \bar{q}_{2}+W_{11}^{(2)}(0) q_{2}\right)\right],
\end{aligned}
$$

where we have used the notation $\boldsymbol{W}_{m n}(\theta)=\left(W_{m n}^{(1)}(\theta), W_{m n}^{(2)}(\theta)\right)^{\mathrm{T}}$. Comparing 2.22) and 2.24), we get

$$
g_{20}=2 p_{1}, g_{11}=p_{2}, g_{02}=2 p_{3}, g_{21}=2 p_{4} .
$$

The next step consists in determining $\boldsymbol{W}_{20}(\theta)$ and $\boldsymbol{W}_{11}(\theta)$, since $g_{21}$ depends on their values. From (2.15) and (2.19), we have

$$
\begin{aligned}
\dot{\boldsymbol{w}}=\dot{\boldsymbol{w}}_{t}-\dot{z} \boldsymbol{q}-\dot{\bar{z}} \overline{\boldsymbol{q}} & = \begin{cases}A \boldsymbol{W}-2 \operatorname{Re}\left\{\overline{\boldsymbol{q}}^{*}(0) \boldsymbol{f}_{0} \boldsymbol{q}(\theta)\right\} & \text { if }-1 \leq \theta<0, \\
A \boldsymbol{W}-2 \operatorname{Re}\left\{\overline{\boldsymbol{q}}^{*}(0) \boldsymbol{f}_{0} \boldsymbol{q}(\theta)\right\}+\boldsymbol{f}_{0} & \text { if } \theta=0\end{cases} \\
& \triangleq A \boldsymbol{W}+\boldsymbol{H}(z, \bar{z}, \theta),
\end{aligned}
$$

where

$$
\boldsymbol{H}(z, \bar{z}, \theta)=\boldsymbol{H}_{20} \frac{z^{2}}{2}+\boldsymbol{H}_{11} z \bar{z}+\boldsymbol{H}_{02} \frac{\bar{z}^{2}}{2}+\cdots
$$

From 2.20), we have

$$
\begin{aligned}
\dot{\boldsymbol{w}}= & \dot{\boldsymbol{w}}_{z} \dot{z}(t)+\dot{\boldsymbol{w}}_{\bar{z}} \dot{\bar{z}}(t) \\
= & \left(\boldsymbol{W}_{20}(\theta) z+\boldsymbol{W}_{11}(\theta) \bar{z}+\cdots\right)\left(\mathrm{i} \omega^{*} \tau_{n}^{*} z(t)+g(z, \bar{z})\right) \\
& +\left(\boldsymbol{W}_{11}(\theta) z+\boldsymbol{W}_{02}(\theta) \bar{z}+\cdots\right)\left(-\mathrm{i} \omega^{*} \tau_{n}^{*} \bar{z}(t)+\bar{g}(z, \bar{z})\right) .
\end{aligned}
$$

It follows from 2.22 that

$$
\begin{aligned}
\dot{w}= & A(0)\left(\boldsymbol{W}_{20}(\theta) \frac{z^{2}}{2}+\boldsymbol{W}_{11}(\theta) z \bar{z}+\boldsymbol{W}_{02}(\theta) \frac{\bar{z}^{2}}{2}+\cdots\right) \\
& +\boldsymbol{H}_{20}(\theta) \frac{z^{2}}{2}+\boldsymbol{H}_{11}(\theta) z \bar{z}+\boldsymbol{H}_{02}(\theta) \frac{\bar{z}^{2}}{2}+\cdots \\
= & \left(A(0) \boldsymbol{W}_{20}(\theta)+\boldsymbol{H}_{20}(\theta)\right) \frac{z^{2}}{2}+\left(A(0) \boldsymbol{W}_{11}(\theta)+\boldsymbol{H}_{11}(\theta)\right) z \bar{z} \\
& +\left(A(0) \boldsymbol{W}_{02}(\theta)+\boldsymbol{H}_{02}(\theta)\right) \frac{\bar{z}^{2}}{2}+\cdots .
\end{aligned}
$$


Comparing the coefficients of $z^{2}$ and $z \bar{z}$ from 2.27 and 2.28 , we get

$$
\left(A(0)-2 \mathrm{i} \omega^{*} \tau_{n}^{*} I\right) \boldsymbol{W}_{20}(\theta)=-\boldsymbol{H}_{20}(\theta), \quad A(0) \boldsymbol{W}_{11}(\theta)=-\boldsymbol{H}_{11}(\theta) .
$$

For $\theta \in[0,1]$, it follows from $2.19,2.20,2.26$ and 2.27 that

$$
\begin{aligned}
\boldsymbol{H}(z, \bar{z}, \theta) & =-\overline{\boldsymbol{q}}^{*}(0) \boldsymbol{f}_{0} \boldsymbol{q}(\theta)-\boldsymbol{q}^{*}(0) \overline{\boldsymbol{f}}_{0} \overline{\boldsymbol{q}}(\theta) \\
& =-g(z, \bar{z}) \boldsymbol{q}(\theta)-\bar{g}(z, \bar{z}) \overline{\boldsymbol{q}}(\theta) \\
& =-\left(g_{20} \frac{z^{2}}{2}+g_{11} z \bar{z}+g_{02} \frac{\bar{z}^{2}}{2}+\cdots\right) \boldsymbol{q}(\theta)-\left(\bar{g}_{20} \frac{\bar{z}^{2}}{2}+\bar{g}_{11} z \bar{z}+\bar{g}_{02} \frac{z^{2}}{2}+\cdots\right) \overline{\boldsymbol{q}}(\theta) .
\end{aligned}
$$

Comparing the coefficients of $z^{2}$ and $z \bar{z}$ between this expression and 2.26 , we get

$$
\boldsymbol{H}_{20}(\theta)=-g_{20} \boldsymbol{q}(\theta)-\bar{g}_{20} \overline{\boldsymbol{q}}(\theta), \quad \boldsymbol{H}_{11}(\theta)=-g_{11} \boldsymbol{q}(\theta)-\bar{g}_{11} \overline{\boldsymbol{q}}(\theta)
$$

From the definition of $A(\theta)$ and 2.29 and 2.30 , we have

$$
\dot{\boldsymbol{W}}_{20}(\theta)=2 \mathrm{i} \omega^{*} \tau_{n}^{*} \boldsymbol{W}_{20}(\theta)+g_{20} \boldsymbol{q}(\theta)+\bar{g}_{02} \overline{\boldsymbol{q}}(\theta)
$$

Since $\boldsymbol{q}(\theta)=\left(q_{1}, q_{2}\right)^{T} \mathrm{e}^{\mathrm{i} \omega^{*} \tau_{n}^{*} \theta}$, we obtain

$$
\boldsymbol{W}_{20}(\theta)=\frac{\mathrm{i} g_{20}}{\omega^{*} \tau_{n}^{*}} \boldsymbol{q}(0) \mathrm{e}^{\mathrm{i} \omega^{*} \tau_{n}^{*} \theta}+\frac{\mathrm{i} \bar{g}_{02}}{3 \omega^{*} \tau_{n}^{*}} \overline{\boldsymbol{q}}(0) \mathrm{e}^{-\mathrm{i} \omega^{*} \tau_{n}^{*} \theta}+\boldsymbol{E}_{1} \mathrm{e}^{2 \mathrm{i} \omega^{*} \tau_{n}^{*} \theta},
$$

where $\boldsymbol{E}_{1}=\left(E_{1}^{(1)}, E_{1}^{(2)}\right)^{\mathrm{T}}$ is a constant vector. Similarly, we have

$$
\boldsymbol{W}_{11}(\theta)=\frac{-\mathrm{i} g_{11}}{\omega^{*} \tau_{n}^{*}} \boldsymbol{q}(0) \mathrm{e}^{\mathrm{i} \omega^{*} \tau_{n}^{*} \theta}+\frac{\mathrm{i} \bar{g}_{11}}{\omega^{*} \tau_{n}^{*}} \overline{\boldsymbol{q}}(0) \mathrm{e}^{-\mathrm{i} \omega^{*} \tau_{n}^{*} \theta}+\boldsymbol{E}_{2},
$$

where $\boldsymbol{E}_{2}=\left(E_{2}^{(1)}, E_{2}^{(2)}\right)^{\mathrm{T}}$ is a constant vector. Now, we shall find the values of $\boldsymbol{E}_{1}$ and $\boldsymbol{E}_{2}$. From the definition of $A(0)$ and (2.31), we have

$$
\begin{gathered}
\int_{-1}^{0} \mathrm{~d} \eta(\theta) \boldsymbol{W}_{20}(\theta)=2 \mathrm{i} \omega^{*} \tau_{n}^{*} \boldsymbol{W}_{20}(0)-\boldsymbol{H}_{20}(0) \\
\int_{-1}^{0} \mathrm{~d} \eta(\theta) \boldsymbol{W}_{11}(\theta)=-\boldsymbol{H}_{11}(0)
\end{gathered}
$$

where $\eta(\theta)=\eta(\theta, 0)$. In view of 2.25, we deduce that when $\theta=0$,

$$
\boldsymbol{H}(z, \bar{z}, 0)-2 \operatorname{Re}\left\{\overline{\boldsymbol{q}}^{*}(0) \boldsymbol{f}_{0} \boldsymbol{q}(0)\right\}+\boldsymbol{f}_{0}=-g(z, \bar{z}) \boldsymbol{q}(0)-\bar{g}(z, \bar{z}) \overline{\boldsymbol{q}}(0)+\boldsymbol{f}_{0}
$$

Then we have

$$
\begin{aligned}
\boldsymbol{H}_{20} \frac{z^{2}}{2}+\boldsymbol{H}_{11} z \bar{z}+\boldsymbol{H}_{02} \frac{\bar{z}^{2}}{2}+\cdots= & -\left(g_{20} \frac{z^{2}}{2}+g_{11} z \bar{z}+g_{02} \frac{\bar{z}^{2}}{2}+\cdots\right) \boldsymbol{q}(0) \\
& -\left(\bar{g}_{20} \frac{\bar{z}^{2}}{2}+\bar{g}_{11} z \bar{z}+\bar{g}_{02} \frac{z^{2}}{2}+\cdots\right) \overline{\boldsymbol{q}}(0)+\boldsymbol{f}_{0} .
\end{aligned}
$$

Comparing both sides of 2.35 , we obtain

$$
\boldsymbol{H}_{20}=-g_{20} \boldsymbol{q}(0)-\bar{g}_{02} \overline{\boldsymbol{q}}(0)+2 \tau_{n}^{*}\left(\begin{array}{c}
H_{1} \\
H_{2}
\end{array}\right), \quad \boldsymbol{H}_{11}=-g_{11} \boldsymbol{q}(0)-\bar{g}_{11} \overline{\boldsymbol{q}}(0)+\tau_{n}^{*}\left(\begin{array}{c}
P_{1} \\
P_{2}
\end{array}\right),
$$

where $H \triangleq\left(H_{1}, H_{2}\right)^{T}$ and $P \triangleq\left(P_{1}, P_{2}\right)^{T}$ are the respective coefficients of $z^{2}$ and $z \bar{z}$ of $\boldsymbol{f}_{0}(z, \bar{z})$. Thus we have

$$
\boldsymbol{H}=\left(\begin{array}{c}
-b_{11}-b_{12} q_{2} \\
-b_{22} q_{2}^{2}+b_{21} q_{2} \mathrm{e}^{-\mathrm{i} \omega^{*} \tau_{n}^{*}}
\end{array}\right), \quad \boldsymbol{P}=\left(\begin{array}{c}
-b_{11}-b_{12} \operatorname{Re}\left\{q_{2}\right\} \\
-b_{22}\left|q_{2}\right|^{2}+b_{21} \operatorname{Re}\left\{\bar{q}_{2} \mathrm{e}^{-\mathrm{i} \omega^{*} \tau_{n}^{*}}\right\}
\end{array}\right) .
$$


Since $\mathrm{i} \omega^{*} \tau_{n}^{*}$ is the eigenvalue of $A(0)$ and $\boldsymbol{q}(0)$ is the corresponding eigenvector, we get

$$
\begin{gathered}
\left(\mathrm{i} \omega^{*} \tau_{n}^{*} I-\int_{-1}^{0} \mathrm{e}^{\mathrm{i} \omega^{*} \tau_{n}^{*} \theta} \mathrm{d} \eta(\theta)\right) \boldsymbol{q}(0)=0 \\
\left(-\mathrm{i} \omega^{*} \tau_{n}^{*} I-\int_{-1}^{0} \mathrm{e}^{-\mathrm{i} \omega^{*} \tau_{n}^{*} \theta} \mathrm{d} \eta(\theta)\right) \overline{\boldsymbol{q}}(0)=0 .
\end{gathered}
$$

Therefore, substituting (2.34) and 2.36) into 2.37), we have

$$
\left(2 \mathrm{i} \omega^{*} \tau_{n}^{*} I-\int_{-1}^{0} \mathrm{e}^{2 \mathrm{i} \omega^{*} \tau_{n}^{*} \theta} \mathrm{d} \eta(\theta)\right) \boldsymbol{E}_{1}=2 \tau_{n}^{*} \boldsymbol{H},
$$

that is $\mathcal{H} \boldsymbol{E}_{1}=2 \boldsymbol{H}$, where

$$
\mathcal{H}=\left[\begin{array}{cc}
2 i \omega^{*}+d_{1} \mu_{i}+b_{11} u_{1}^{*} & b_{12} u_{1}^{*} \\
-b_{21} u_{2}^{*} \mathrm{e}^{-\mathrm{i} \omega^{*} \tau_{n}^{*}} & 2 \mathrm{i} \omega^{*}+d_{2} \mu_{i}+b_{22} u_{2}^{*}
\end{array}\right] .
$$

Thus, according to Cramer's rule, $E_{1}^{(i)}=2 \Delta_{i} / \Delta$, where $\Delta=\operatorname{det} \mathcal{H}$ and $\Delta_{i}=\operatorname{det} \mathcal{U}_{i}$, where $\mathcal{U}_{i}$ is formed by replacing the $i$-th column vector of $\mathcal{H}$ by another column vector $\left(H_{1}, H_{2}\right)^{\mathrm{T}}$ for $i=1,2$. In a similar way, we have $\mathcal{P} \boldsymbol{E}_{2}=2 \boldsymbol{P}$, where

$$
\mathcal{P}=\left[\begin{array}{cc}
d_{1} \mu_{i}+b_{11} u_{1}^{*} & b_{12} u_{1}^{*} \\
-b_{21} u_{2} & d_{2} \mu_{i}+b_{22} u_{2}^{*}
\end{array}\right] .
$$

Thus $E_{2}^{(i)}=2 \tilde{\Delta}_{i} / \tilde{\Delta}$, where $\tilde{\Delta}=\operatorname{det} \mathcal{P}$ and $\tilde{\Delta}_{i}=\operatorname{det} \mathcal{V}_{i}$, where $\mathcal{V}_{i}$ is formed by replacing the $i$-th column vector of $\mathcal{P}$ by another column vector $\left(P_{1}, P_{2}\right)^{\mathrm{T}}$ for $i=1,2$. Therefore, we can determine $\boldsymbol{W}_{20}(\theta)$ and $\boldsymbol{W}_{11}(\theta)$ from 2.32 and 2.33 . Furthermore, we can easily compute $g_{21}$. Again, according to 28, the Hopf bifurcation periodic solutions of 1.1$)$ at $\tau_{n}^{*}$ on the center manifold are determined by the following formulas:

$$
\begin{aligned}
C_{1}(0) & =\frac{\mathrm{i}}{2 \tau_{n}^{*} \omega^{*}}\left(g_{11} g_{20}-2\left|g_{11}\right|^{2}-\frac{\left|g_{02}\right|^{2}}{3}+\frac{g_{21}}{2}\right), \\
\nu_{2} & =-\operatorname{Re}\left\{C_{1}(0)\right\}\left(\operatorname{Re}\left\{\frac{\mathrm{d} \lambda}{\mathrm{d} \tau}\left(\tau_{n}^{*}\right)\right\}\right)^{-1}, \\
\beta_{2} & =2 \operatorname{Re}\left\{C_{1}(0)\right\}, \\
T_{2} & =\frac{1}{\tau_{n}^{*} \omega^{*}}\left(-\operatorname{Im}\left\{C_{1}(0)\right\}+\nu_{2} \operatorname{Im}\left\{\frac{\mathrm{d} \lambda}{\mathrm{d} \tau}\left(\tau_{n}^{*}\right)\right\}\right) .
\end{aligned}
$$

Here $\nu_{2}$ determines the direction of the Hopf bifurcation. If $\nu_{2}>0\left(\nu_{2}<0\right.$, respectively $)$ then these bifurcations are supercritical (subcritical, respectively) and the bifurcating periodic solutions exist for $\tau>\tau_{n}^{*}\left(\tau<\tau_{n}^{*}\right.$, respectively). Once again $\beta_{2}$ determines the stability of the bifurcating periodic solutions: they are stable (unstable) if $\beta_{2}<0\left(\beta_{2}>0\right.$, respectively). Also, $T_{2}$ determines their period: the period increases (decreases, respectively) if $T_{2}>0\left(T_{2}<0\right.$, respectively). We collect these results in the following theorem.

Theorem 2.6. Assume that $\operatorname{Re}\left\{C_{1}(0)\right\}<0\left(\operatorname{Re}\left\{C_{1}(0)\right\}>0\right.$, respectively). Then,

- the Hopf bifurcation of solutions to (1.1) at the equilibrium $\boldsymbol{u}^{*}$ when $\tau=\tau_{n}^{*}$ is supercritical (subcritical, respectively), and

- the bifurcating periodic solutions on the center manifold are stable (unstable, respectively). 


\subsection{Global stability of the equilibrium}

First we study the global stability of the equilibrium when the delay is absent.

Theorem 2.7. If (1.1) satisfies (2.4) and we choose $\tau=0$, then its positive equilibrium $\boldsymbol{u}^{*}$ is globally asymptotically stable.

Proof We define $E(t)=E_{1}(t)+E_{2}(t)$, where

$$
E_{i}(t):=\int_{\Omega} b_{3-i, i}\left[u_{i}-u_{i}^{*}-u_{i}^{*} \log \left(\frac{u_{i}}{u_{i}^{*}}\right)\right] \mathrm{d} \boldsymbol{x}, \quad i=1,2 .
$$

We now show that $E(t)$ is a Lyapunov functional. It is obvious that $E(t) \geq 0$. We now prove that $\mathrm{d} E(t) / \mathrm{d} t \leq 0$. Differentiating $E_{1}(t)$, replacing $\partial_{t} u_{1}$ by using $1.1 \mathrm{a}$ and employing the homogeneous Neumann boundary condition $(1.1 \mathrm{c})$, we get

$$
\dot{E}_{1}(t)=-b_{21} d_{1} u_{1}^{*} \int_{\Omega} \frac{\left|\nabla u_{1}\right|^{2}}{u_{1}^{2}} \mathrm{~d} \boldsymbol{x}-b_{21} \int_{\Omega}\left(u_{1}-u_{1}^{*}\right)\left(b_{11}\left(u_{1}-u_{1}^{*}\right)+b_{12}\left(u_{2}-u_{2}^{*}\right)\right) \mathrm{d} \boldsymbol{x} .
$$

Analogously, we obtain

$$
\dot{E}_{2}(t)=-b_{12} d_{2} u_{2}^{*} \int_{\Omega} \frac{\left|\nabla u_{2}\right|^{2}}{u_{2}^{2}} \mathrm{~d} \boldsymbol{x}+b_{12} \int_{\Omega}\left(u_{2}-u_{2}^{*}\right)\left(b_{21}\left(u_{1}-u_{1}^{*}\right)-b_{22}\left(u_{2}-u_{2}^{*}\right)\right) \mathrm{d} \boldsymbol{x} .
$$

Combining the expressions for $\dot{E}_{1}(t)$ and $\dot{E}_{2}(t)$, we have

$$
\begin{aligned}
\dot{E}(t)= & -b_{21} d_{1} u_{1}^{*} \int_{\Omega} \frac{\left|\nabla u_{1}\right|^{2}}{u_{1}^{2}} \mathrm{~d} \boldsymbol{x}-b_{12} d_{2} u_{2}^{*} \int_{\Omega} \frac{\left|\nabla u_{2}\right|^{2}}{u_{2}^{2}} \mathrm{~d} \boldsymbol{x}-b_{11} b_{21} \int_{\Omega}\left(u_{1}-u_{1}^{*}\right)^{2} \mathrm{~d} \boldsymbol{x} \\
& -b_{22} b_{12} \int_{\Omega}\left(u_{2}-u_{2}^{*}\right)^{2} \mathrm{~d} \boldsymbol{x} \leq 0 .
\end{aligned}
$$

This completes the proof.

Now we study the global stability of the equilibrium in the presence of delay.

Theorem 2.8. If the system (1.1) satisfies the assumption (2.4) and

$$
\max \left\{b_{12}, b_{21}\right\} \leq\left(b_{11} b_{22}\right)^{1 / 2},
$$

then the positive equilibrium $\boldsymbol{u}^{*}$ of (1.1) is globally asymptotically stable.

Proof. We now define $F(t)=F_{1}(t)+F_{2}(t)+F_{3}(t)$, where

$$
\begin{aligned}
& F_{i}(t):=\int_{\Omega} b_{3-i, i}\left[u_{i}-u_{i}^{*}-u_{i}^{*} \log \left(\frac{u_{i}}{u_{i}^{*}}\right)\right] \mathrm{d} \boldsymbol{x}, \quad i=1,2, \\
& F_{3}(t):=\int_{\Omega} \int_{t-\tau}^{t} \frac{b_{11}}{2}\left(u_{1}(s, \boldsymbol{x})-u_{1}^{*}\right)^{2} \mathrm{~d} s \mathrm{~d} \boldsymbol{x} .
\end{aligned}
$$

It is obvious that $F(t) \geq 0$. In a similar way as for Theorem 2.7 we show that $\dot{F} \leq 0$. First, direct computation yields

$$
\begin{aligned}
& \dot{F}_{1}(t)=-d_{1} u_{1}^{*} \int_{\Omega} \frac{\left|\nabla u_{1}\right|^{2}}{u_{1}^{2}} \mathrm{~d} \boldsymbol{x}-\int_{\Omega}\left(u_{1}-u_{1}^{*}\right)\left(b_{11}\left(u_{1}-u_{1}^{*}\right)+b_{12}\left(u_{2}-u_{2}^{*}\right)\right) \mathrm{d} \boldsymbol{x}, \\
& \dot{F}_{2}(t)=-d_{2} u_{2}^{*} \int_{\Omega} \frac{\left|\nabla u_{2}\right|^{2}}{u_{2}^{2}} \mathrm{~d} \boldsymbol{x}+\int_{\Omega}\left(u_{2}-u_{2}^{*}\right)\left(b_{21}\left(u_{1}(t-\tau, \boldsymbol{x})-u_{1}^{*}\right)-b_{22}\left(u_{2}-u_{2}^{*}\right)\right) \mathrm{d} \boldsymbol{x},
\end{aligned}
$$




$$
\dot{F}_{3}(t)=\frac{b_{11}}{2} \int_{\Omega}\left(\left(u_{1}-u_{1}^{*}\right)^{2}-\left(u_{1}(t-\tau, \boldsymbol{x})-u_{1}^{*}\right)^{2}\right) \mathrm{d} \boldsymbol{x} .
$$

Combining these equations, we obtain

$$
\begin{aligned}
\dot{F}(t)= & -d_{1} u_{1}^{*} \int_{\Omega} \frac{\left|\nabla u_{1}\right|^{2}}{u_{1}^{2}} \mathrm{~d} \boldsymbol{x}-d_{2} u_{2}^{*} \int_{\Omega} \frac{\left|\nabla u_{2}\right|^{2}}{u_{2}^{2}} \mathrm{~d} \boldsymbol{x} \\
& -\int_{\Omega}\left[\frac{b_{11}}{2}\left(u_{1}-u_{1}^{*}\right)^{2}+b_{22}\left(u_{2}-u_{2}^{*}\right)^{2}+\frac{b_{11}}{2}\left(u_{1}(t-\tau, \boldsymbol{x})-u_{1}^{*}\right)^{2}\right] \mathrm{d} \boldsymbol{x} \\
& +\int_{\Omega}\left[b_{21}\left(u_{1}(t-\tau, \boldsymbol{x})-u_{1}^{*}\right)\left(u_{2}-u_{2}^{*}\right)-b_{12}\left(u_{1}-u_{1}^{*}\right)\left(u_{2}-u_{2}^{*}\right)\right] \mathrm{d} \boldsymbol{x} .
\end{aligned}
$$

Thus, in view of (2.38), it follows that $\dot{F}(t) \leq 0$. Therefore, $F(t)$ is a Lyapunov functional and the proof is completed.

Remark 2.1. Theorem 2.8 implies that if 2.38 holds, then the time delay can not induce spatial patterns.

\section{Numerical method}

\subsection{Finite volume element spatial discretization}

Let $\mathscr{T}_{h}$ be a family of partitions of $\Omega$ into triangles $K$, and assume that $\mathscr{T}_{h}$ with meshsize $h>0$ is regular in the sense of [15. By $\mathbb{P}^{r}(K)$ we denote the space of polynomial functions on $K \in \mathscr{T}_{h}$ of degree at most $r \geq 0$. The classical finite dimensional space of continuous piecewise linear functions

$$
V_{h}=\left\{v_{h} \in C^{0}(\bar{\Omega}):\left.v_{h}\right|_{K} \in \mathbb{P}^{1}(K), \forall K \in \mathscr{T}_{h}\right\},
$$

spanned by the Lagrangian basis $\left\{\phi_{i}\right\}_{i}$ of linear nodal functions (and satisfying $\phi_{i}\left(s_{j}\right)=\delta_{j i}$ for the vertices $s_{j}$ of $\mathscr{T}_{h}$, where $\delta_{j i}$ is the Kronecker delta) is a subspace of $H^{1}(\Omega)$ used to write the weak formulation in a semi-discrete sense:

For $t>0$, find $u_{1, h}(t), u_{2, h}(t) \in V_{h}$ such that

$$
\begin{aligned}
& \frac{\mathrm{d}}{\mathrm{d} t}\left(u_{1, h}(t), v_{h}\right)_{\Omega}+d_{1}\left(\nabla u_{1, h}(t), \nabla v_{h}\right)_{\Omega}=\left(f_{1, h}(t), v_{h}\right)_{\Omega} \quad \forall v_{h} \in V_{h}, \\
& \frac{\mathrm{d}}{\mathrm{d} t}\left(u_{2, h}(t), w_{h}\right)_{\Omega}+d_{2}\left(\nabla u_{2, h}(t), \nabla w_{h}\right)_{\Omega}=\left(f_{2, h}(t), w_{h}\right)_{\Omega} \quad \forall w_{h} \in V_{h},
\end{aligned}
$$

where the nonlinear reaction terms in the semi-discrete setting are given by

$$
\begin{aligned}
f_{1, h}(t) & :=u_{1, h}(t)\left(a_{1}-b_{11} u_{1, h}(t)-b_{12} u_{2, h}(t)\right), \\
f_{2, h}(t, \tau) & :=u_{2, h}(t)\left(-a_{2}+b_{21} u_{1, h}(\tau)-b_{12} u_{2, h}(t)\right) .
\end{aligned}
$$

Existence and uniqueness of solutions to (3.1) along with a-priori estimates can be obtained following [43.

The associated finite volume element discretization is obtained by introducing a dual mesh $\mathscr{T}_{h}^{*}$ consisting of polygons (control volumes) $K_{i}^{*}$ centered on each node $s_{i}$ of $\mathscr{T}_{h}$ and defined by joining the barycenter of each element sharing the vertex $s_{i}$ and the midpoints of the edges that intersect $s_{i}$, see Figure 1 .

We next define the finite volume space $V_{h}^{*}$ as

$$
V_{h}^{*}:=\left\{w \in L^{2}(\Omega): w_{K_{i}^{j}} \in \mathbb{P}^{0}\left(K_{i}^{*}\right), \forall K_{i}^{*} \in \mathscr{T}_{h}^{*}\right\},
$$




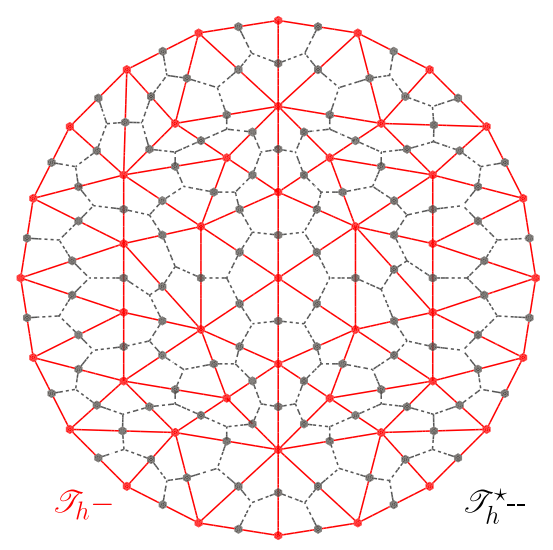

Figure 1: Sketch of a primal mesh $\mathscr{T}_{h}$ (solid lines) and a dual mesh $\mathscr{T}_{h}^{*}$ (dashed lines) of a disk-shaped domain.

and a Petrov-Galerkin map $\mathcal{P}_{h}: V_{h} \rightarrow V_{h}^{\star}$ (cf., e.g., [46]) defined by

$$
\left(\mathcal{P}_{h} v_{h}\right)(x)=\sum_{i} v_{h}\left(s_{i}\right) \chi_{i}(x) \quad \text { for } x \in \Omega,
$$

where $\chi_{i}$ is the characteristic function on the control volume $K_{i}^{\star} \in \mathscr{T}_{h}^{*}$, that is, $\chi_{i}(x)=1$ if $x \in K_{i}^{\star}$ and zero otherwise. Using Lemma 3.1 of [35], we end up with a semi-discrete finite volume element formulation where functions of the dual test space $V_{h}^{*}$ appear in the mass terms associated to the time derivatives and the nonlinear reaction kinetics:

$$
\begin{aligned}
& \text { For } t>0, \text { find } u_{1, h}(t), u_{2, h}(t) \in V_{h} \text { such that } \\
& \frac{\mathrm{d}}{\mathrm{d} t}\left(u_{1, h}(t), \mathcal{P}_{h} v_{h}\right)_{\Omega}+d_{1}\left(\nabla u_{1, h}(t), \nabla v_{h}\right)_{\Omega}=\left(f_{1, h}(t), \mathcal{P}_{h} v_{h}\right)_{\Omega} \quad \forall v_{h} \in V_{h}, \\
& \frac{\mathrm{d}}{\mathrm{d} t}\left(u_{2, h}(t), \mathcal{P}_{h} w_{h}\right)_{\Omega}+d_{2}\left(\nabla u_{2, h}(t), \nabla w_{h}\right)_{\Omega}=\left(f_{2, h}(t, \tau), \mathcal{P}_{h} w_{h}\right)_{\Omega} \quad \forall w_{h} \in V_{h} .
\end{aligned}
$$

The experimental spatial convergence properties of the method will be addressed in Section 4.2 .

\subsection{Runge-Kutta time discretization}

A fully discrete method is obtained after partitioning the time interval $\mathcal{T}$ into nodes $\left\{t^{k}\right\}_{k=-m}^{N}$, where $t^{0}=0, t^{-m}=\tau$ and $t^{N}=T$; and choosing a stable Runge-Kutta time integration scheme 32] (see also [3] for tests on the robustness and performance of RK methods for differential delay equations). The resulting system of equations is

$$
\begin{aligned}
\frac{1}{\Delta t} \mathbb{L} \boldsymbol{Y}_{i}^{n}+\sum_{j=1}^{i} \alpha_{i j} \mathbb{K} \boldsymbol{Y}_{j}^{n} & =\frac{1}{\Delta t} \mathbb{L} \boldsymbol{U}^{n}+\sum_{j=1}^{i-1} \mathbb{L} \tilde{\alpha}_{i j} \boldsymbol{F}\left(\boldsymbol{Y}_{j}^{n}, \boldsymbol{Y}_{j}^{n-m}\right), \quad i=1, \ldots, s, \\
\frac{1}{\Delta t} \mathbb{L} \boldsymbol{U}^{n+1}+\sum_{i=1}^{s} \beta_{i} \mathbb{K} \boldsymbol{Y}_{i}^{n} & =\frac{1}{\Delta t} \mathbb{L} \boldsymbol{U}^{n}+\sum_{i=1}^{s} \mathbb{L} \tilde{\beta}_{i} \boldsymbol{F}\left(\boldsymbol{Y}_{i}^{n}, \boldsymbol{Y}_{i}^{n-m}\right),
\end{aligned}
$$

where $s$ is the order of the RK scheme, $\boldsymbol{U}$ is the vector of nodal values of the discrete solution $\left(u_{1, h}(t), u_{2, h}(t)\right), \boldsymbol{Y}_{i}$ is the vector of the discrete solution in the intermediate stage $i \in\{1, \ldots, s\}, \mathbb{L}$ is the primal-dual projection matrix (between $V_{h}$ and $V_{h}^{\star}$ ) with entries $\int_{\Omega} \phi_{i} \chi_{j} \mathrm{~d} \boldsymbol{x}, \mathbb{K}$ is the stiffness matrix with entries $\int_{\Omega} \nabla \phi_{i} \cdot \nabla \phi_{j} \mathrm{~d} \boldsymbol{x}$, and $\boldsymbol{F}\left(\boldsymbol{A}^{n}, \boldsymbol{A}^{n-m}\right)$ is the vector of reaction terms depending on the discrete generic field $\boldsymbol{A}$ at times $t=t^{n}$ and $t=t^{n}-\tau=t^{n-m}$. System (3.2) is solved at each RK step using a generalized minimal residual (GMRES) method with a fixed tolerance. 
(a)

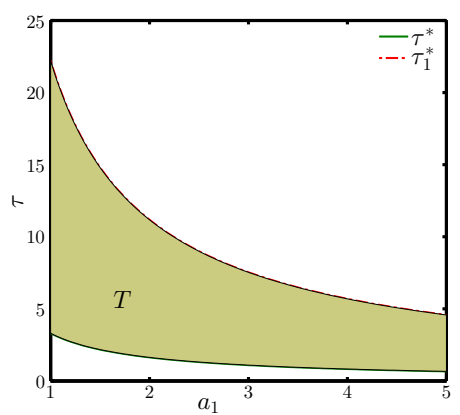

(b)

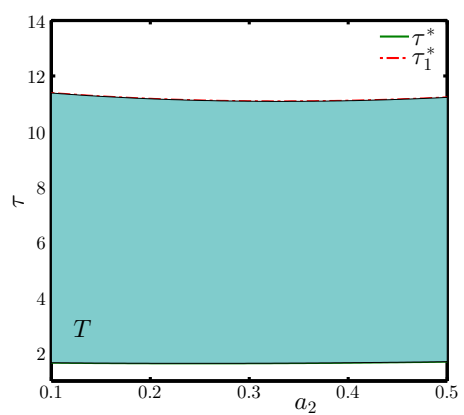

(c)

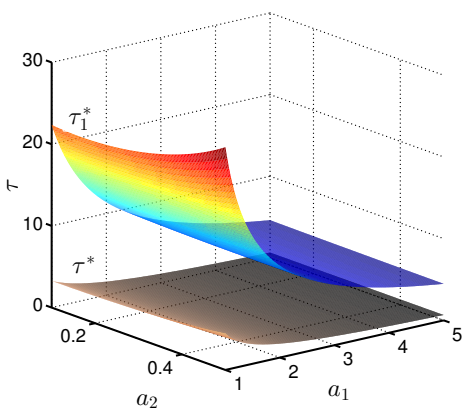

Figure 2: Bifurcation diagram of model 1.1 for parameters $a_{1}$ (a), $a_{2}$ (b), and $a_{1}, a_{2}$ (c), where the remaining parameters are fixed to $b_{11}=0.2, b_{12}=0.5, b_{21}=0.2, b_{22}=0.2, d_{1}=0.1, d_{2}=0.1$. The figures show the Hopf instability space (labeled as $T$ in plots $(\mathrm{a}, \mathrm{b})$ ), corresponding to the region bounded by the two Hopf bifurcations $\tau=\tau^{*}=\tau_{0}^{*}$ and $\tau=\tau_{1}^{*}$.

Note that the FVE method in 35] (combined with a backward difference formula of order 2 for the time discretization) actually assumed $\frac{\mathrm{d}}{\mathrm{d} t}\left(u_{1, h}(t), \mathcal{P}_{h} v_{h}\right)_{\Omega} \approx \frac{\mathrm{d}}{\mathrm{d} t}\left(u_{1, h}(t), v_{h}\right)_{\Omega}$, and analogously for the second species $w$. In relation with 3.2 this suggests that, in the terms multiplied by $\frac{1}{\Delta t}$, the coefficient matrix $\mathbb{L}$ could be replaced by the mass matrix $\mathbb{M}$ with entries $\int_{\Omega} \phi_{i} \phi_{j} \mathrm{~d} \boldsymbol{x}$. However, such an approximation may generate larger errors, especially in the case of multistep methods. The convergence behaviour of the time discretization has been predicted by the results in [10, and will be confirmed numerically in the tests of Section 4.2 .

\section{Numerical results}

\subsection{Hopf parameter space}

In view of Theorem 2.4. satisfaction of condition 2.4 is sufficient for the positive uniform equilibrium $\left(u_{1}^{*}, u_{2}^{*}\right)$ to be linearly unstable with respect to the particular case of system (1.1). In the simulations we will take the following values in the parameter space:

$$
a_{1}=2, a_{2}=0.2, b_{11}=0.2, b_{12}=0.5, b_{21}=0.2, b_{22}=0.2, d_{1}=0.1, d_{2}=0.1 .
$$

For this particular choice, the positive uniform equilibrium is given by

$$
\left(u_{1}^{*}, u_{2}^{*}\right)=\left(\frac{25}{7}, \frac{18}{7}\right) \approx(3.5714,2.5714)
$$

Now, we present the bifurcations represented by the formula 2.11) in the parameter region spanned by the parameters $a_{1}$ and $\tau$ that are also depicted in Figure 2. All arising spatial patterns are induced in this parameter region.

Following a standard procedure (cf., e.g., [42]), we can compute the wavenumber explicitly and characterize the pattern selection mechanism by the linearization around the positive uniform equilibrium and taking $\tau$ as the Hopf bifurcation parameter. From the mathematical viewpoint, the Hopf bifurcation occurs when $\operatorname{Im}(\lambda)>0, \operatorname{Re}(\lambda)=0$ and $\mathrm{d} \operatorname{Re}(\lambda(\tau)) / \mathrm{d} \tau>0$ at $\sqrt{\mu_{i}}$ for some fixed $i$. By the eigenvalue theory of the parabolic differential equations, $k_{c}:=\sqrt{\mu_{i}}$ is the critical wavenumber and $\lambda$ is the root of the characteristic equation (2.6).

We comment that the solution behaviour of (1.1) depends on the choice of the parameters and on the shape and the size of the computational domain. Consider, for instance, a square-shaped 
domain $\Omega=(0, L)^{2}$. The eigenvalues $\mu_{i}$ are given by

$$
\mu_{i}=\frac{\pi^{2}}{L^{2}} s_{i}, \quad i \in \mathbb{N},
$$

where we assume that $\left\{s_{i}\right\}_{i \in \mathbb{N}} \subset \mathbb{N}$ is a strictly increasing list of all numbers of the form $s_{i}=m^{2}+n^{2}$, $m, n \in \mathbb{N}_{0}$, i.e., $s_{1}=0, s_{2}=1, s_{3}=2, s_{4}=4, s_{5}=5, s_{6}=8$, etc. Note that independently of $L$, we have $Q=0.9184$, and that $\mu_{1}=0$ independently of the shape and size of the domain. We here consider square-shaped domains with $L=60$ or $L=2 \pi$, and the resulting coefficients are reported in Table 1.

\begin{tabular}{ccccccc}
\hline \hline$i$ & $s_{i}$ & $\mu_{i}$ & $R_{i}$ & $\omega^{*}$ & $\tau^{*}$ & $\tau_{1}^{*}$ \\
\hline \multicolumn{6}{c}{ Results for $L=60$} \\
\hline 1 & 0 & 0.0000 & 1.2285 & 0.6587 & 1.6372 & 11.1749 \\
2 & 1 & 0.0027 & 1.2291 & 0.6585 & 1.6382 & 11.1785 \\
3 & 2 & 0.0054 & 1.2296 & 0.6584 & 1.6391 & 11.1821 \\
4 & 4 & 0.0109 & 1.2307 & 0.6580 & 1.6409 & 11.1894 \\
5 & 5 & 0.0137 & 1.2313 & 0.6578 & 1.6419 & 11.1930 \\
6 & 8 & 0.0219 & 1.2329 & 0.6572 & 1.6446 & 11.2039 \\
\hline \multicolumn{6}{c}{ Results for $L=2 \pi$} \\
\hline 1 & 0 & 0.0000 & 1.2285 & 0.6587 & 1.6372 & 11.1749 \\
2 & 1 & 0.2500 & 1.2785 & 0.6419 & 1.7221 & 11.5103 \\
3 & 2 & 0.5000 & 1.3285 & 0.6254 & 1.8081 & 11.8545 \\
4 & 4 & 1.0000 & 1.4285 & 0.5936 & 1.9826 & 12.5667 \\
5 & 5 & 1.2500 & 1.4785 & 0.5784 & 2.0709 & 12.9334 \\
6 & 8 & 2.0000 & 1.6285 & 0.5356 & 2.3391 & 14.0685 \\
\hline
\end{tabular}

Table 1: Coefficients leading to the formation of patterns on the square domain $\Omega=(0, L)^{2}$, for two different sizes.

\subsection{Example 1: error histories for a simplified model}

The accuracy of the numerical method is first studied for the reduced system

$$
\partial_{t} u_{1}-\Delta u_{1}=-\left(u_{1}\right)_{\tau}+\frac{u_{2}}{2}, \quad \partial_{t} u_{2}-\Delta u_{2}=-2\left(u_{1}\right)_{\tau}+u_{2},
$$

defined on $\Omega=(0,2 \pi)^{2}, t \in[0,1]$ with homogeneous Neumann boundary conditions and data $u_{1}(x, y, t)=\cos (x)+\cos (y), u_{2}(x, y, t)=2(\cos (x)+\cos (y))$ for $t \in[-\tau, 0]$. Its exact solution is $u_{1}=w(t)[\cos (x)+\cos (y)], u_{2}=2 u_{1}$ where

$$
w(t)= \begin{cases}1 & \text { if }-\tau \leq t<0, \\ 1-t & \text { if } 0 \leq t<1, \\ \frac{1}{2}(t-1)^{2} & \text { if } 1 \leq t<2\end{cases}
$$

is a solution of the following delayed ordinary differential equation:

$$
\frac{\mathrm{d} w}{\mathrm{~d} t}=-w(t-\tau) \quad \text { for } t \in(0, \infty) ; \quad w(t)=1 \quad \text { for } t \in[-\tau, 0]
$$

An RK scheme of order $s=2$ will be used, with parameters $\alpha_{21}=0, \alpha_{22}=1, \tilde{\alpha}_{21}=1, \tilde{\alpha}_{22}=0$, $\beta_{1}=0, \beta_{2}=1, \tilde{\beta}_{1}=1, \tilde{\beta}_{2}=0$ (corresponding to an implicit RK method, see [32]). When required, the exact solution (if available) is employed as additional initial data. Otherwise, we employ the homogeneous equilibrium state perturbed with a uniformly distributed random field. 


\begin{tabular}{|ccccc|ccccc|}
\hline \multicolumn{6}{|c|}{ Spatial accuracy test } & \multicolumn{5}{c|}{ Temporal accuracy test } \\
$h$ & $E_{0}\left(u_{1}\right)$ & rate & $E_{0}\left(u_{2}\right)$ & rate & $\Delta t$ & $e_{1}\left(u_{1}\right)$ & rate & $e_{1}\left(u_{2}\right)$ & rate \\
\hline 4.4428 & 2.3512 & - & 3.7025 & - & $1.4921 \mathrm{e}-3$ & 6.1668 & - & 12.3322 & - \\
2.2214 & $6.7035 \mathrm{e}-1$ & 1.9104 & 1.3415 & 1.8143 & $7.5455 \mathrm{e}-4$ & $9.6801 \mathrm{e}-1$ & 1.9732 & 1.9368 & 1.9735 \\
1.1107 & $1.5941 \mathrm{e}-1$ & 2.0685 & $3.1974 \mathrm{e}-1$ & 2.0683 & $3.7564 \mathrm{e}-4$ & $2.5238 \mathrm{e}-1$ & 1.8150 & $5.0914 \mathrm{e}-1$ & 1.8151 \\
0.5553 & $3.9429 \mathrm{e}-2$ & 2.0198 & $7.8853 \mathrm{e}-2$ & 2.0199 & $1.8477 \mathrm{e}-4$ & $6.7396 \mathrm{e}-2$ & 1.8898 & $1.3329 \mathrm{e}-1$ & 1.8928 \\
0.2776 & $9.8244 \mathrm{e}-3$ & 2.0040 & $1.9620 \mathrm{e}-2$ & 2.0037 & $9.3753 \mathrm{e}-5$ & $1.3442 \mathrm{e}-2$ & 1.9552 & $2.6925 \mathrm{e}-2$ & 1.9551 \\
0.1388 & $2.4576 \mathrm{e}-3$ & 1.9994 & $4.9153 \mathrm{e}-3$ & 1.9994 & $4.6841 \mathrm{e}-5$ & $3.5837 \mathrm{e}-3$ & 1.9818 & $7.3106 \mathrm{e}-3$ & 1.9822 \\
0.0694 & $6.1743 \mathrm{e}-4$ & 1.9996 & $1.2332 \mathrm{e}-3$ & 1.9995 & $2.3412 \mathrm{e}-5$ & $8.1670 \mathrm{e}-4$ & 1.9854 & $1.6343 \mathrm{e}-3$ & 1.9849 \\
\hline
\end{tabular}

Table 2: Example 1: convergence histories for the FVE-RK approximation of the reduced system 4.2.

(a)

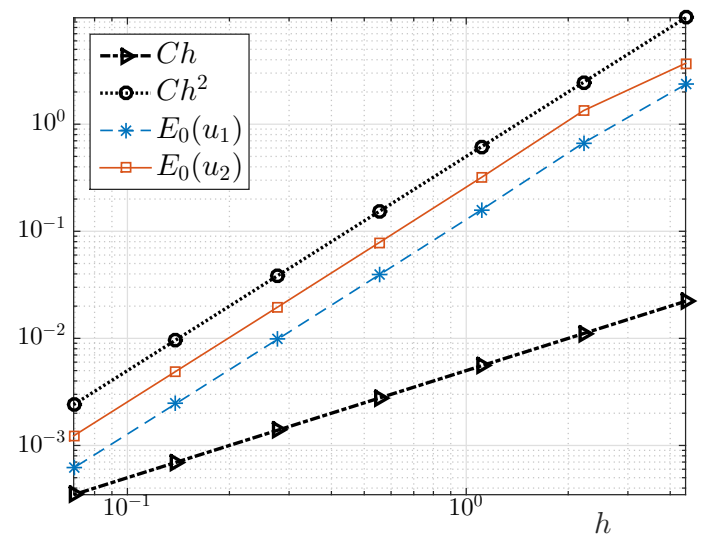

(b)

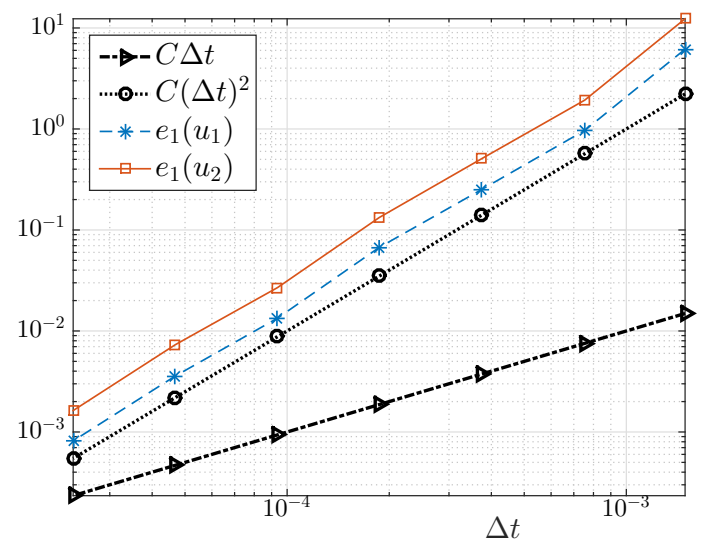

Figure 3: Example 1: errors $E_{0}\left(u_{1}\right), E_{0}\left(u_{2}\right)$ versus the meshsize $\left(\right.$ a) and $e_{1}(\cdot), e_{0}(\cdot)$ versus the timestep $(\mathrm{b})$ associated to the FVE-RK approximation of the reduced system 4.2 . See values in Table 2

The observed convergence rates of the approximate solutions are illustrated by computing errors in the $L^{2}\left(0, T ; H^{1}(\Omega)\right)$-norm and at the final time $T=1$ in the $L^{2}(\Omega)$-norm, defined as

$$
e_{1}\left(u_{i}\right):=\left(\Delta t \sum_{n=-m}^{N}\left\|u_{i}\left(t^{n}\right)-u_{i, h}^{n}\right\|_{H^{1}(\Omega)}^{2}\right)^{1 / 2}, \quad E_{0}\left(u_{i}\right):=\left\|u_{i}\left(t^{N}\right)-u_{h, i}^{N}\right\|_{L^{2}(\Omega)},
$$

for $i=1,2$. The spatial accuracy is assessed by a series of computations on a sequence of structured triangular primal meshes where each node of a coarser mesh is also present in a finer mesh and the timestep is first fixed to $\Delta t=10^{-5}$ so that the total error is dominated by the spatial component of the error. Secondly, we study the time accuracy of the RK scheme by fixing $h=0.0694$ and running a set of tests with nested timesteps. We put $\tau=0.25$ and the results can be observed in Figure 3 and Table 2, where we report on the errors and experimental orders of convergence of $(\Delta t)^{2}$ for $e_{1}\left(u_{i}\right)$ and of $h^{2}$ for $E_{0}\left(u_{i}\right)$.

\subsection{Example 2: full delayed predator-prey model on a square}

We continue with simulations for system (1.1) modeling a predator-prey scenario where the domain of interest is the square $\Omega=(0, L)^{2}$ with $L=60$. The corresponding wavenumber satisfies

$$
\boldsymbol{k}=\pi\left(m_{1} / L, m_{2} / L\right), \quad|\boldsymbol{k}|=\pi \sqrt{\left(m_{1} / L\right)^{2}+\left(m_{2} / L\right)^{2}}, \quad \text { for } m_{1}, m_{2}=0,1, \ldots
$$


(a)

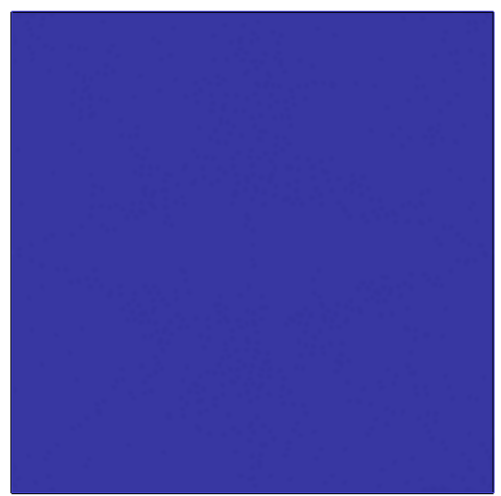

(c)

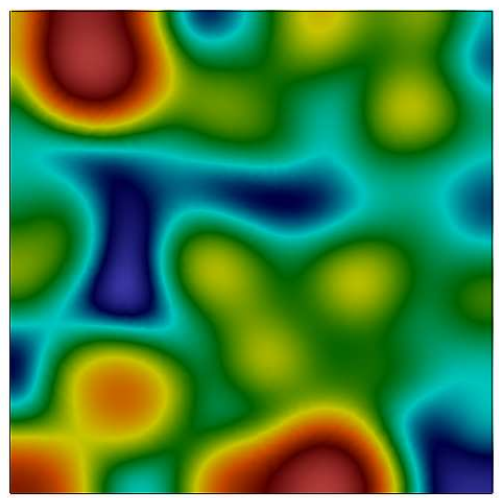

(e)

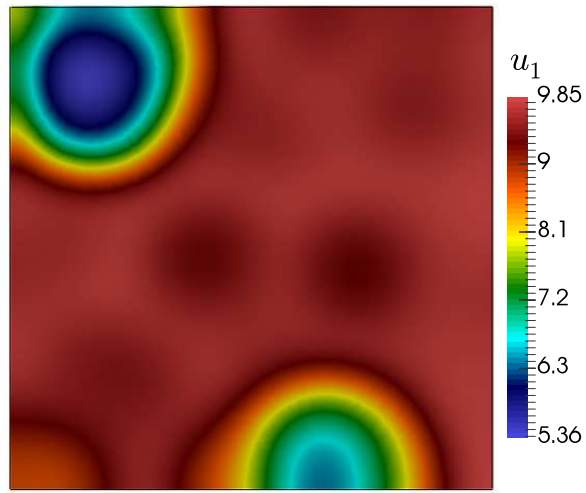

(b)

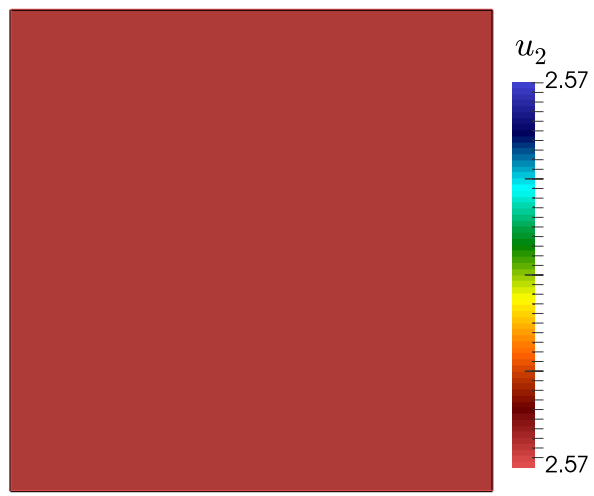

(d)

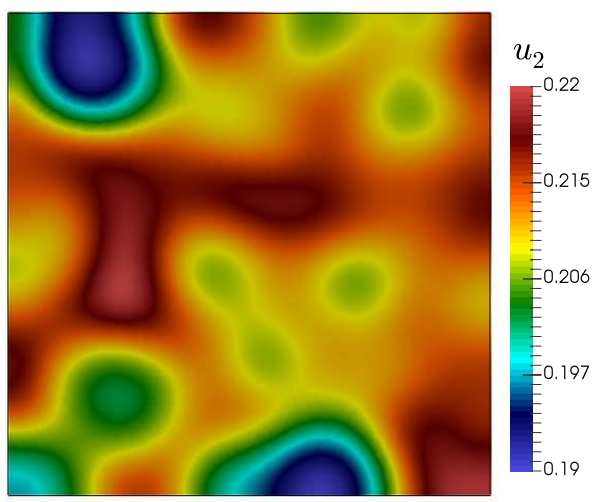

(f)

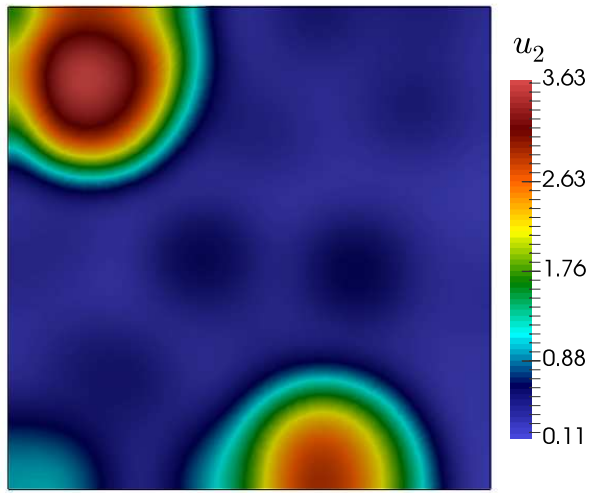

Figure 4: Example 2: spatial patterns of species $u_{1}$ (a,c,e) and $u_{2}$ (b,d,f) for different time delays $\tau=1.5(\mathrm{a}, \mathrm{b}), \tau=5$ $(\mathrm{c}, \mathrm{d})$, and $\tau=12(\mathrm{e}, \mathrm{f})$. Snapshots correspond to $t=1000$.

The timestep size is chosen as $\Delta t=10^{-3}$ and the system is evolved until $T=1000$. Here and in Examples 3 to 5, the numerical solutions start from certain noisy initial patterns such that the bounds for the exact solution, 2.2 and 2.3 in Theorem 2.3 , are actually given by

$$
0 \leq u_{1}(\boldsymbol{x}, t) \leq \mathcal{A}_{1}=\frac{a_{1}}{b_{11}}=10, \quad 0 \leq u_{2}(\boldsymbol{x}, t) \leq \mathcal{A}_{2}=\frac{1}{b_{22}}\left(\frac{b_{21} a_{1}}{b_{11}}-a_{2}\right)=9 .
$$


(a)

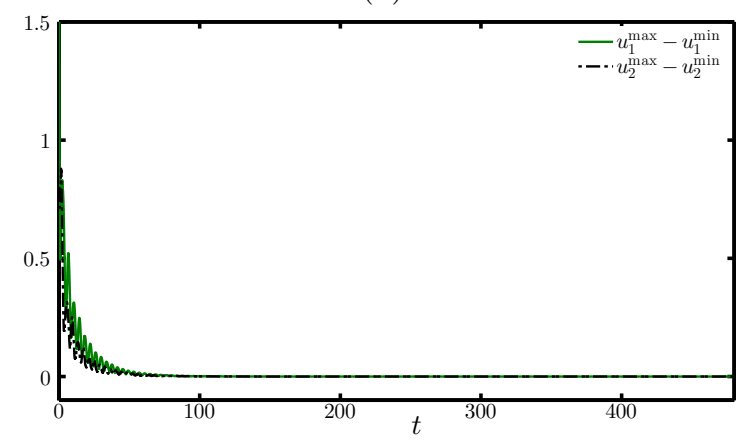

(c)

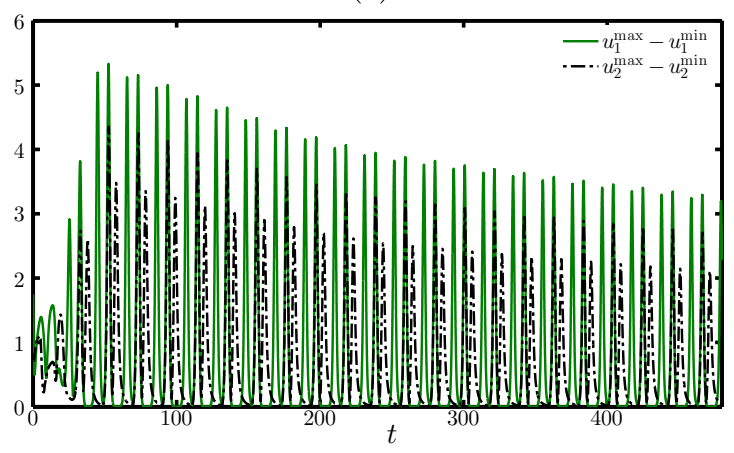

(e)

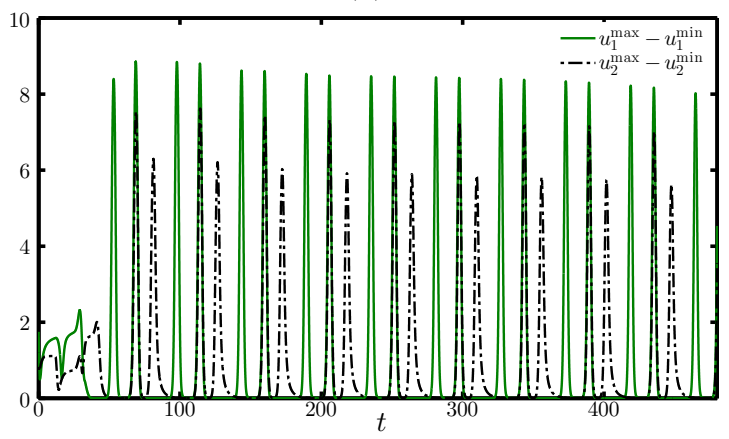

(b)

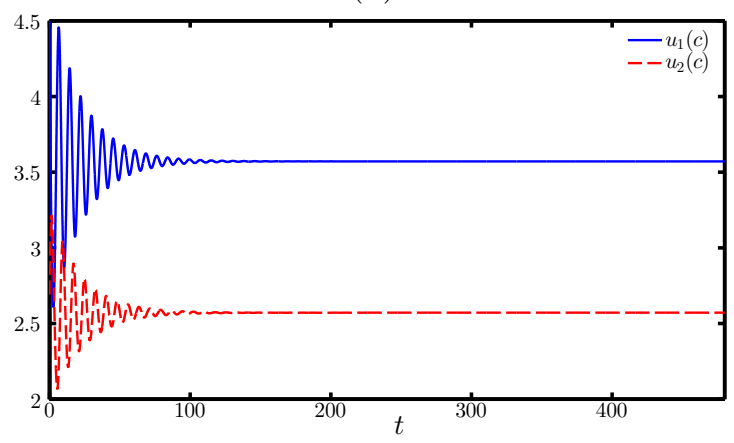

(d)

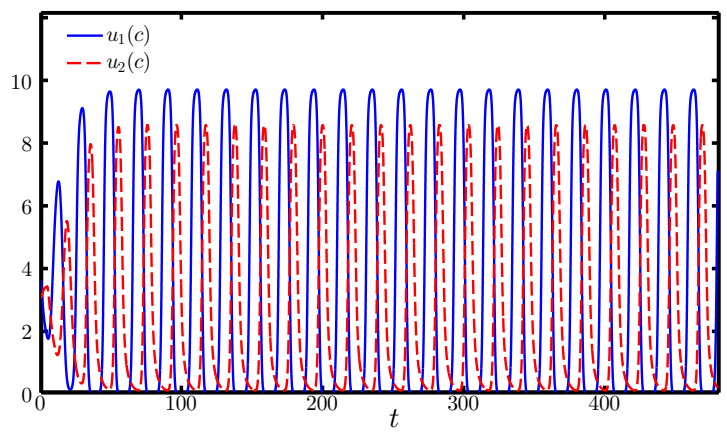

(f)

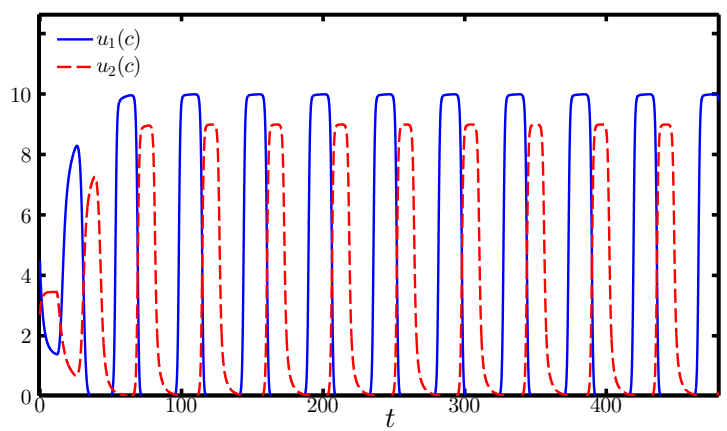

Figure 5: Example 2: time evolution of the total variation (a,c,e) and density of species on the center $c$ of the domain (b,d,f) up to $t=480$ for different time delays $\tau=1.5(\mathrm{a}, \mathrm{b}), \tau=5(\mathrm{c}, \mathrm{d})$, and $\tau=12(\mathrm{e}, \mathrm{f})$. When $\tau<\tau^{*}=1.6373$, the initial patterns are smoothed out. If $\tau>\tau^{*}$, regular patterns appear with a given frequency, and if $\tau>\tau_{1}^{*}=11.1749$, patterns form with a frequency twice the previous one. These simulations agree with the sketch in Figure 2

Apart from those given in Section 4.1, different combinations of model parameters have been successfully tested indicating the significance of the model under various scenarios. From (2.11) we compute the Hopf bifurcation thresholds $\tau^{*}=1.637276$ and $\tau_{1}^{*}=11.174933$ corresponding to $s_{1}=0$, and an example for different values of $\tau$ is presented in Figures 4 and 5 . Periodic solutions should appear due to the Hopf bifurcation. In addition, when the time delay $\tau$ is less than the critical value $\tau^{*}=1.637276$, no patterns are generated. For instance, in the top-right plot of Figure 5 corresponding to the time evolution of the solution at the center of the domain and for $\tau=1.5<\tau^{*}$, we see that the initial patterns are smoothed out. From the theoretical results we expect that for $1.8<\tau<11.2$ the amplitude of the oscillations will increase significantly within a short period. This is confirmed when we put $\tau=5$, where we can observe the formation of patterns. This is also the case if we set the time delay as $\tau=\Delta t+\tau^{*}$. We finally put $\tau=12$ and notice that spatial 
(a)

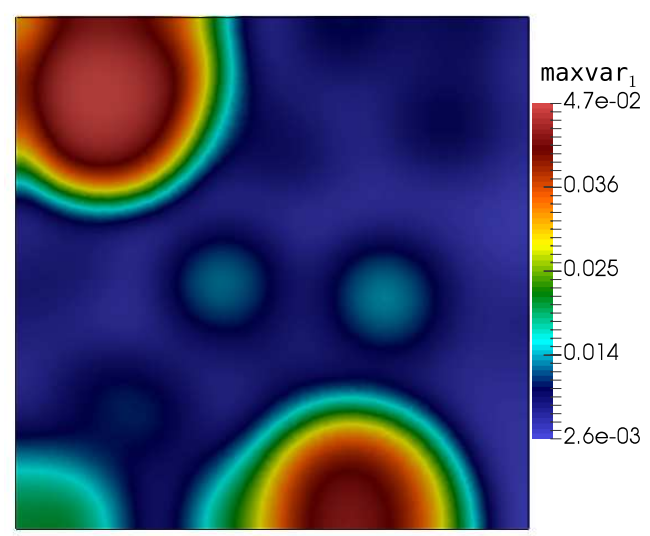

(b)

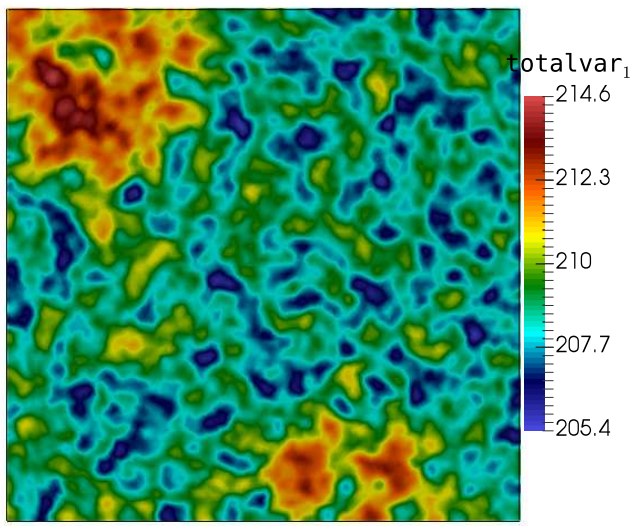

Figure 6: Example 2: maximum (a) and total (b) variations through time for species $u_{1}$ (similar patterns are observed for $u_{2}$ and therefore not shown).

patterns appear within a period of around $2 \tau$. This can be especially noticed in the bottom-left plot of Figure 5. A similar behaviour is observed for a delay $\tau=\Delta t+\tau_{1}^{*}$. A primal mesh with 38952 elements and 19733 vertices has been employed. The average number of GMRES iterations needed to achieve convergence with a tolerance of $10^{-7}$ was 8 . Note that in this example and in Examples 3 to 5 , the numerical solution for $u_{1}$ and $u_{2}$ assumes values in the intervals $[0,10]$ and $[0,9]$, respectively, in agreement with (4.3). This property provides further support of the numerical method.

We compute the fields of maximum and total variations for the species $u_{j}, j=1,2$ at a given point $\boldsymbol{x} \in \Omega$ in $t \in[-\tau, T]$ as

$$
\begin{aligned}
\operatorname{maxvar}_{j}(\boldsymbol{x}) & :=\max _{-m \leq k \leq N} u_{j, h}^{k}(\boldsymbol{x})-\min _{-m \leq k \leq N} u_{j, h}^{k}(\boldsymbol{x}), \\
\operatorname{totalv_{j}}(\boldsymbol{x}) & :=\sum_{k=1-m}^{N}\left|u_{j, h}^{k}(\boldsymbol{x})-u_{j, h}^{k-1}(\boldsymbol{x})\right|,
\end{aligned}
$$

respectively. These quantities are shown for $\tau=12$ in Figure 6 .

\subsection{Example 3: predator-prey model with large delays}

In this third example we introduce an initial noisy pattern in the solution and observe the evolution of these patterns in Figure 7 . Here the domain is $(0,2 \pi)^{2}$, the time delay is $\tau=50$, and we study the behaviour of the system until $t=400=8 \tau$. From Figure 8 we see that the spatial patterns present in the initial datum remain visible over a longer period of time than in Example 2. However, the numerical solution displayed in Figure 7) especially the plots for $t=200=4 \tau$ and $t=250=5 \tau$, indicates that the solution eventually assumes the behaviour of a synchronous oscillation (that is, both species exhibit the same cyclic pattern) over the whole domain. This becomes visible in the fact that the range of $u_{1, h}$ is from 8.55 to 9.79 at $t=200=4 \tau$, which is fairly close to $\mathcal{A}_{1}=10$, while at $t=250=5 \tau$, solution values are practically identically zero. However, the solution component $u_{1, h}$ shown in Figure 7 (f) does not remain identically zero. In fact, as can be inferred from Figure 8 (b), $u_{1, h}$ returns to values close to $\mathcal{A}_{1}$ shortly after $t=300=6 \tau$. Moreover, the plot of $u_{1}^{\max }-u_{1}^{\min }$ (Figure 8 (a)) exhibits a "peak" centered at roughly $t=310$ with $u_{1}^{\max }-u_{1}^{\min } \approx 0$ for extended time intervals before and after that peak. This behaviour indicates that the numerical solution is practically constant on the whole domain except for very short time intervals. Analogous statements 
(a)

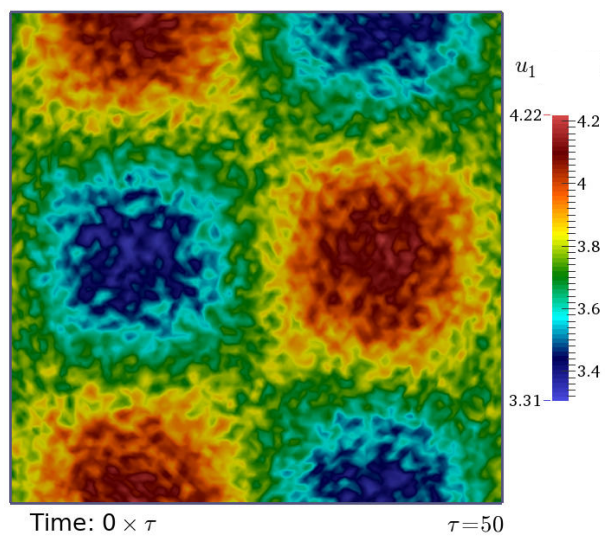

(c)

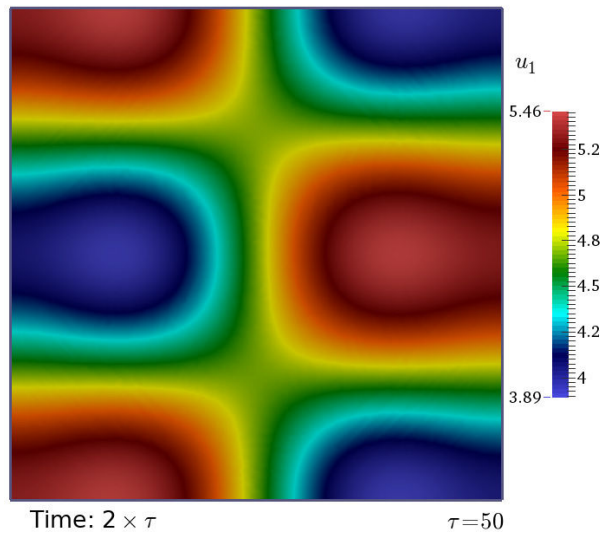

(e)

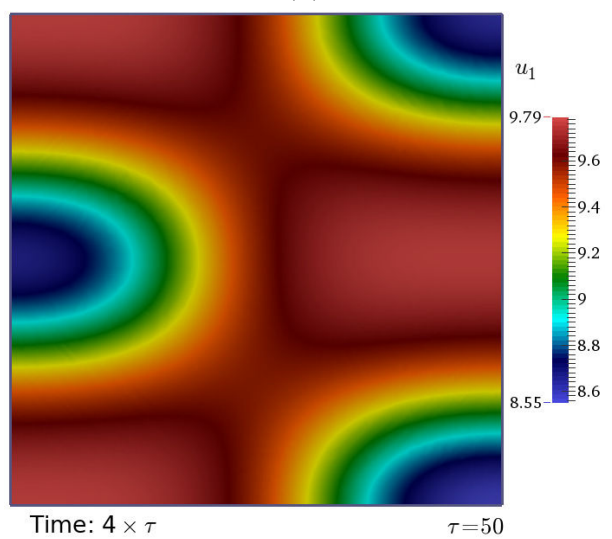

(b)

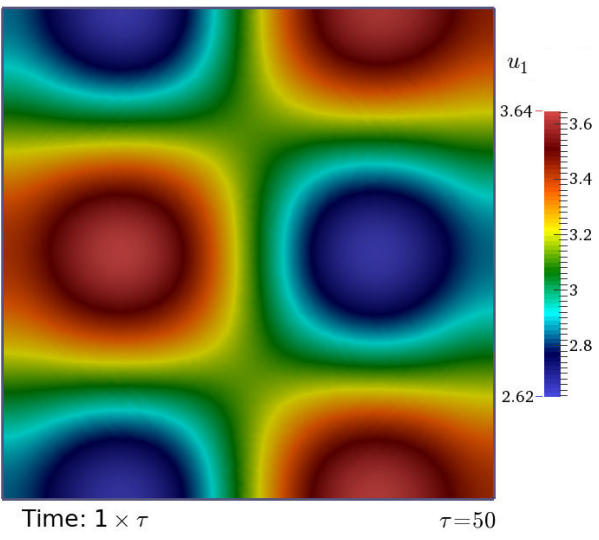

(d)

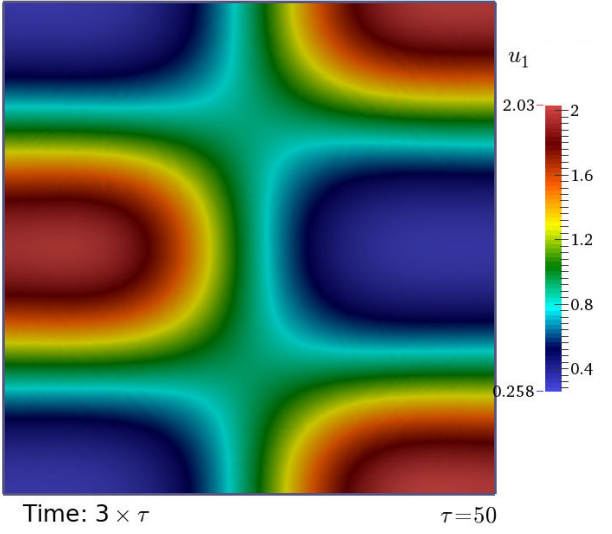

(f)

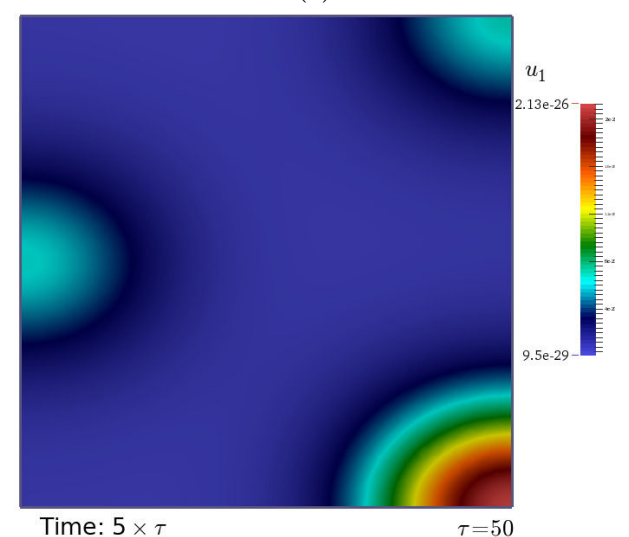

Figure 7: Example 3: evolution of spatial patterns of species $u_{1}$ on the square $(0,2 \pi)^{2}$ for a time delay of $\tau=50$. Snapshots going from $t=0$ (a) to $t=5 \tau$ (f).

correspond to $u_{2, h}$, for which results are not displayed. The solution behaviour is consistent with the observation that there are no stable states like $\left(u_{1}^{*}, 0\right)$ or $\left(0, u_{2}^{*}\right)$, as can be deduced from the analysis of Section 2.3 (especially, considering (2.6-2.8) under the assumption $u_{2}^{*}=0$ or $u_{1}^{*}=0$. 
(a)

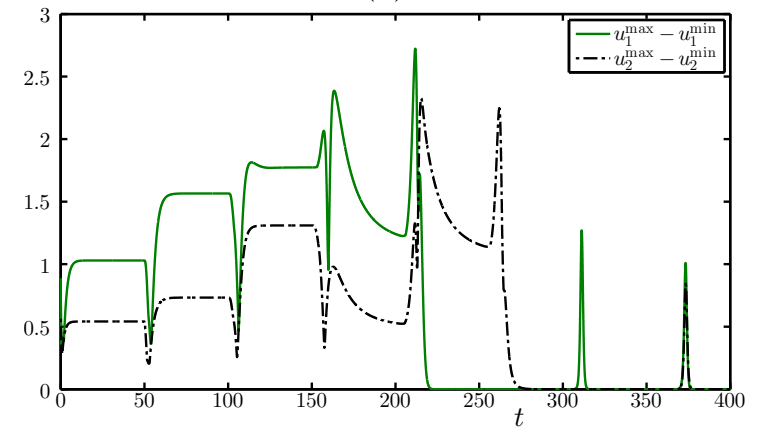

(b)

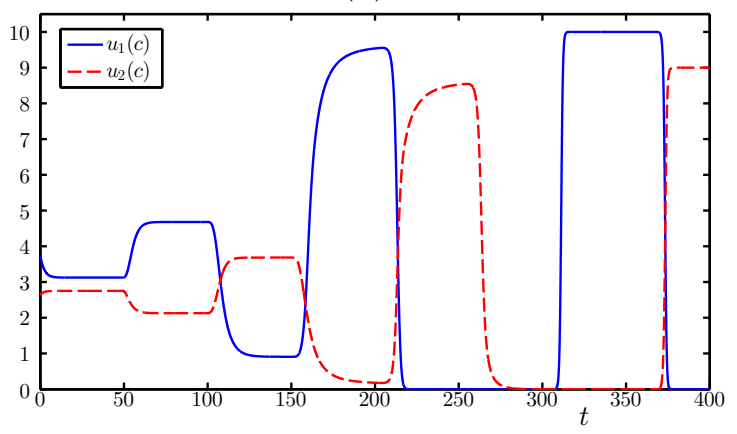

Figure 8: Example 3: dynamics of the maximum and total variations (a) and density of species on the domain center $c$ (b) for a time delay of $\tau=50$.

\subsection{Example 4: full delayed predator-prey model on a disk}

The patterns can be observed with further detail in a simulation with a smaller wavenumber. The next simulation is then performed on a disk of radius $r=10$ and Figures 9 illustrate the behaviour of the model for species $u_{1}$ at time instants $t=24$ and $t=480$. The patterns developed for species $u_{2}$ exhibit a similar spatial distribution (but with different values) as those arising for species $u_{1}$. These plots show that when the delay $\tau$ is close to $\tau_{1}^{*}$, the pattern modes are different than those arising when $\tau$ is close to $\tau^{*}$. In this way we are able to numerically reproduce two critical points for the formation of spatial patterns. According to Theorem 2.5. we could obtain, in fact, more than two critical points.

\subsection{Example 5: formation of spiral waves in a full delayed predator-prey model}

For our last example we simulate the formation of spiral waves in a rectangular domain $\Omega=$ $(0,900) \times(0,300)$. The initial conditions are taken as small perturbation of the equilibrium state $u_{1}^{0}(x, y)=u_{1}^{*}-\epsilon_{1}(x-y / 10-225)(x-y / 10-675), u_{2}^{0}(x, y)=u_{2}^{*}-\epsilon_{2}(x-445)-\epsilon_{3} *(y-150)$, at two given points $(91.4634,201.2195)$ and $(684.1463,245.1219)$ where the species densities are zero. We choose $\epsilon_{1}=2 \mathrm{e}-7, \epsilon_{2}=3 \mathrm{e}-5, \epsilon_{3}=1.2 \mathrm{e}-4$. This datum will simultaneously trigger the formation of two spiral waves whose amplitudes will increase with time and eventually will propagate into the whole domain. Three snapshots at times $t=20,100,200$ are shown in Figure 10. They indicate that although the initial datum is chosen as a small perturbation around $\boldsymbol{u}^{*}$, for each fixed time the numerical solution for $u_{1}$ and $u_{2}$ assumes values that are close to zero on some parts of the domain and close to $\mathcal{A}_{1}$ and $\mathcal{A}_{2}$, respectively, on almost all other parts, with both regions separated by a relatively sharp boundary. Moreover, the numerical results indicate an oscillation in time, and in contrast to Examples 2 to 4, there is no visible tendency towards a synchronous en bloc oscillation on the whole domain.

\section{Conclusion}

In this paper we have presented the theoretical formulation, consistent mathematical analysis, and numerical implementation of pattern formation phenomena in a predator-prey model with delay terms. Applying a stability analysis and suitable numerical simulations, we investigate the Hopf parameter space, the Hopf bifurcation and the pattern selection. We have shown that the time delay can lead to the formation of spatio-temporal patterns when the carrying capacity of the prey is large. The stability of the positive uniform equilibrium is determined in the Hopf parameter space. 
(a)

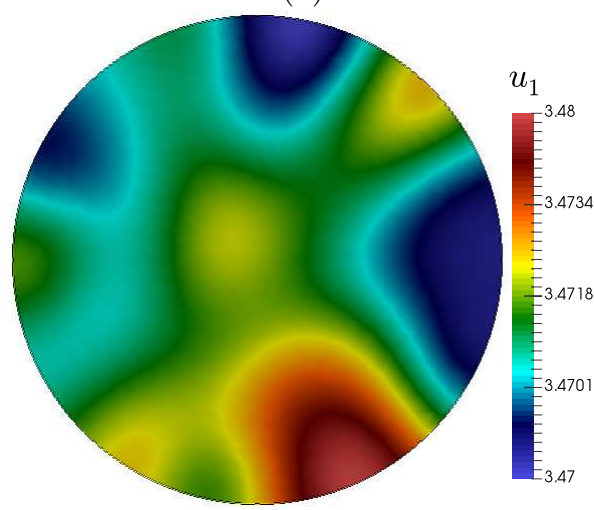

(c)

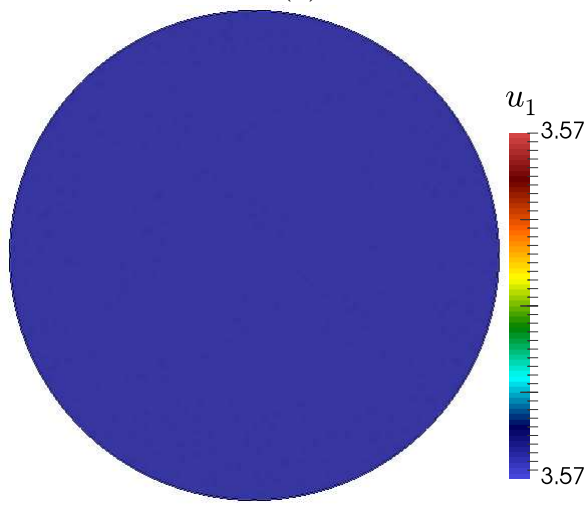

(e)

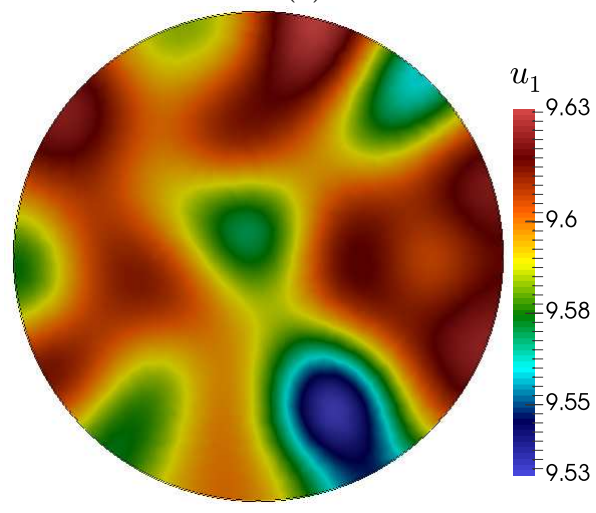

(b)

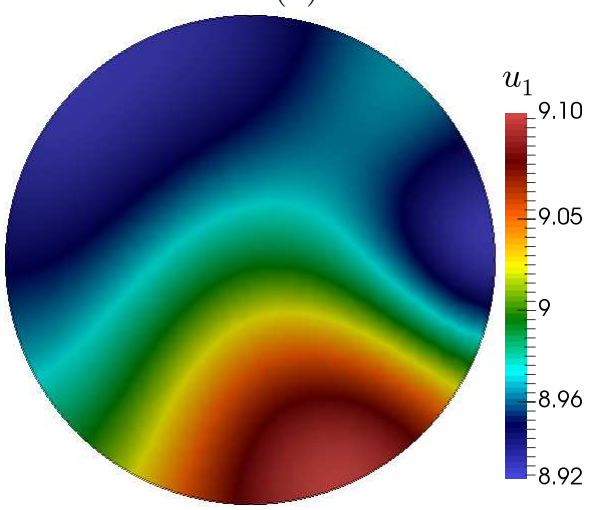

(d)

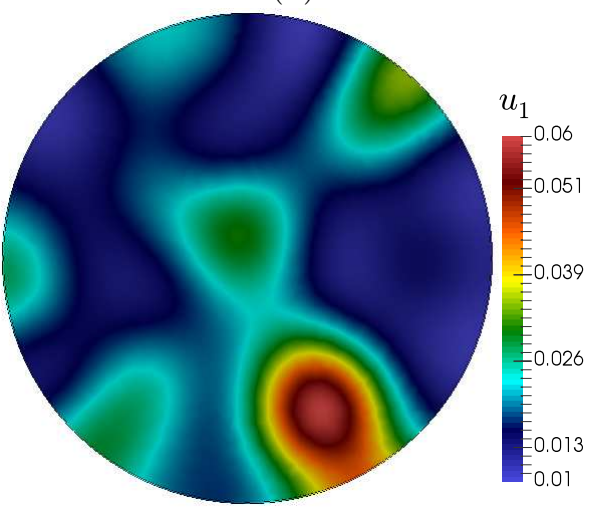

(f)

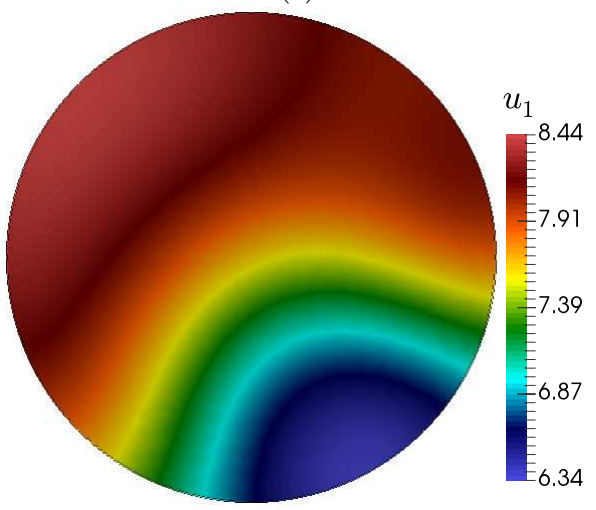

Figure 9: Example 4: snapshots of the transient patterns of species $u_{1}$ at $t=24(\mathrm{a}, \mathrm{c}, \mathrm{e})$ and $t=480(\mathrm{~b}, \mathrm{~d}, \mathrm{f})$ for time delays $\tau=1.5(\mathrm{a}, \mathrm{b}), \tau=5(\mathrm{c}, \mathrm{d})$, and $\tau=12(\mathrm{e}, \mathrm{f})$.

The existence of stability switches induced by the delay is found in the region of the Hopf space. Coming back to the original biological interpretation of (1.1), we find that the explicit inclusion of delay as a gestation period or reaction time may give rise to complex spatio-temporal behaviour. In view of Examples 2 to 5 it is, in particular, remarkable that when the delay assumes a supercritical value, either distinct spatial patterns form (as in Example 5) or both population densities become nearly constant on the whole domain, and change almost abruptly between zero and the respective maximum possible value. Finally, we mention that among other advantages, the numerical scheme 
(a)

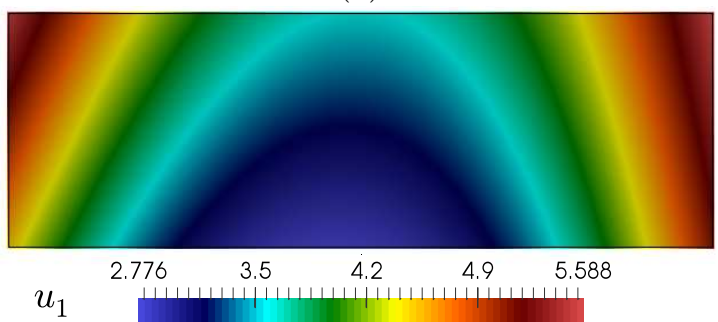

(c)

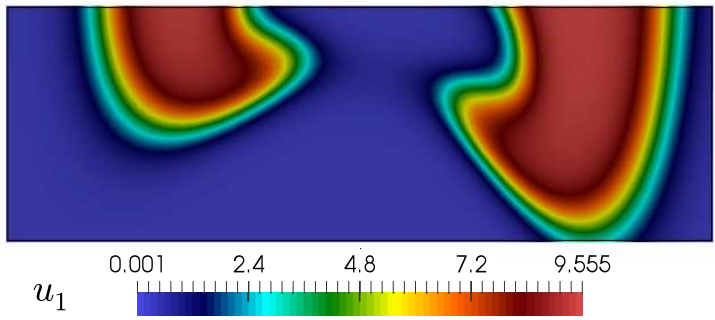

(e)

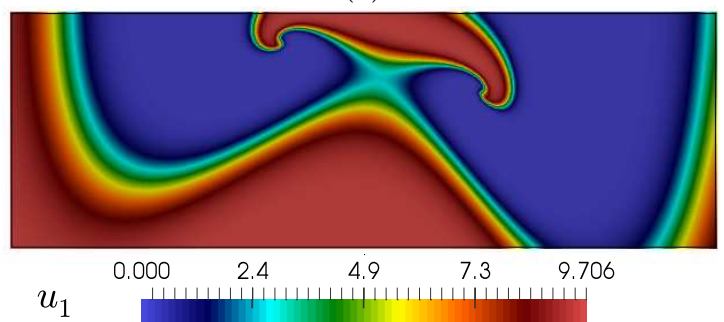

(b)

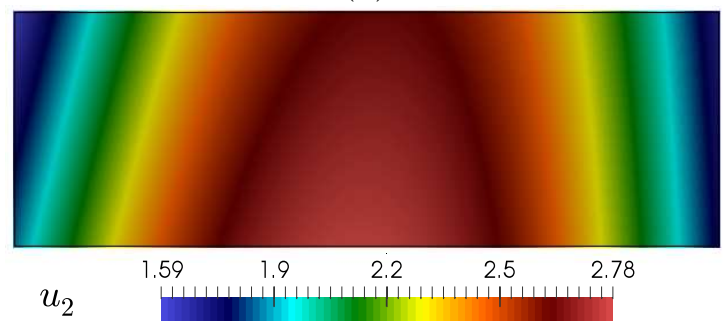

(d)

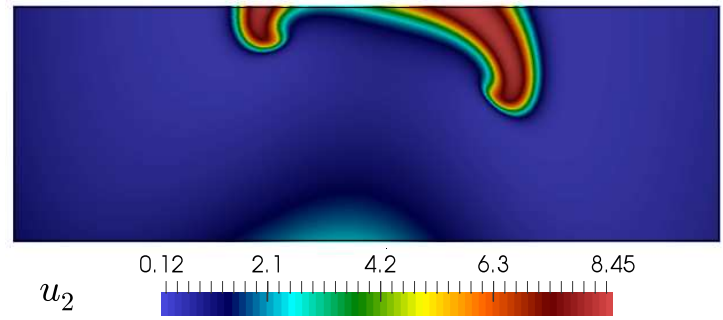

(f)

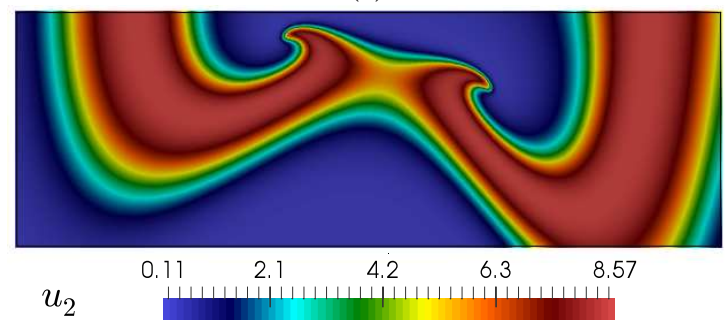

Figure 10: Example 5: snapshots of the transient patterns of species $u_{1}(\mathrm{a}, \mathrm{c}, \mathrm{e})$ and $u_{2}(\mathrm{~b}, \mathrm{~d}, \mathrm{f})$ at times $t=20$ (a,b), $t=100(\mathrm{c}, \mathrm{d})$, and $t=200(\mathrm{e}, \mathrm{f})$, for a time delay of $\tau=5$.

is not bound to a Cartesian grid, and therefore can handle real biological domains that are usually shaped somewhat irregularly.

Numerical studies have been employed to support and extend the obtained theoretical results. The numerical simulations illustrate the existence of both stable and unstable equilibrium near the critical point of the delay which is in good agreement with our theoretical analysis results. Our result does not cover yet the case of time delay far away from the critical point. At the current stage the present model is still quite simple and can of course accommodate a number of directions for improvement. For instance, the description of the prey-predator by a bilinear function, namely the term $f\left(u_{1}, u_{1}\right):=b_{12} u_{1} u_{2}$ in 1.1a), corresponds to a simple biological situation and works well for small density of the prey, that is, when the carrying capacity $a_{1} / b_{11}$ is big, as is assumed in ths paper (cf., e.g., (2.4)). However, it has been argued hat such a model is not realistic and should be replaced by a prey-predator interaction given by a nonlinear concave saturation function such as the Holling type II functional response $f\left(u_{1}, u_{2}\right)=b_{12} u_{1} u_{2} /\left(A+u_{1}\right)$, where $A>0$ is constant (see, e.g., 7, Ch. 5] or [36, Ch. 4] for details and alternative choices). While it would certainly be interesting to attempt to extend the Hopf bifurcation analysis of Section 2 to a version of (1.1) that includes such an alternative functional response and the steps required for this purpose are clear, let us comment that the results of Section 2 for the present model depend in an involved way on the specific algebraic structure of the right-hand sides of $1.1 \mathrm{a}$ and $1.1 \mathrm{~b}$ and it is not obvious, for instance, whether one arrives at the same possibilities for spatio-temporal pattern formation for alternative models. That said, we comment that the numerical scheme introduced in Section 3 can 
easily be adapted to alternative functional responses.

\section{Acknowledgement}

RB acknowledges support by Fondecyt project 1130154; BASAL project CMM, Universidad de Chile and Centro de Investigación en Ingeniería Matemática $\left(\mathrm{CI}^{2} \mathrm{MA}\right)$, Universidad de Concepción; and CONICYT project Anillo ACT1118 (ANANUM). RRB acknowledges support by the Elsevier Mathematical Sciences Sponsorship Fund MSSF-201602. CT acknowledges partial support by the PRC grant NSFC 11201406 and the Qinglan Project. We are very grateful to two anonymous referees for valuable comments that resulted in a number of improvements of the paper.

\section{References}

[1] B. Andreianov, M. Bendahmane, R. Ruiz-Baier, Analysis of a finite volume method for a cross-diffusion model in population dynamics, Math. Models Methods Appl. Sci. 21(02) (2011) 307-344.

[2] A. Bellen, M. Zennaro, Numerical Methods for Delay Differential Equations, Clarendon Press, Oxford University Press, New York, 2003.

[3] G. Bencheva, Comparative analysis of solution methods for delay differential equations in hematology. In: LSSC 2009, Lirkov, I. et al., eds., Lect. Notes Comput. Sci. vol. 5910, Springer-Verlag, New York, $2010,711-718$.

[4] M. Bendahmane, V. Anaya, M. Sepúlveda, Mathematical and numerical analysis for predator-prey system in a polluted environment, Netw. Heter. Media 5(4) (2010) 813-847.

[5] M. Bendahmane, R. Ruiz-Baier, C. Tian, Turing pattern dynamics and adaptive discretization of a superdiffusive Lotka-Volterra system, J. Math. Biol. 72 (2016) 1441-1465.

[6] J.M. Bownds, J.M. Cushing, On the behaviour of solutions of predator-prey equations with hereditary terms, Math. Biosci. 26 (1975) 41-54.

[7] F. Brauer, C. Castillo-Chávez, Mathematical Models in Population Biology and Epidemiology, Second Ed., Springer-Verlag, New York, 2012.

[8] R. Bürger, S. Kumar, R. Ruiz-Baier, Discontinuous finite volume element discretization for coupled flow-transport problems arising in models of sedimentation, J. Comput. Phys. 299 (2015) 446-471.

[9] R. Bürger, R. Ruiz-Baier, H. Torres, A stabilized finite volume element formulation for sedimentationconsolidation processes, SIAM J. Sci. Comput. 34 (2012) B265-B289.

[10] E. Burman, A. Ern, Implicit-explicit Runge-Kutta schemes and finite elements with symmetric stabilization for advection-diffusion equations, ESAIM: Math. Model. Numer. Anal. 46 (2012) 681-707.

[11] Z. Cai, On the finite volume element method, Numer. Math. 58 (1991) 713-735.

[12] S.H. Chou, Analysis and convergence of a covolume method for the generalized Stokes problem, Math. Comput. 66 (1997) 85-104.

[13] S.-N. Chow, J.K. Hale, Methods of Bifurcation Theory, Springer-Verlag, New York, 1982.

[14] M. Cross, H. Greenside, Pattern Formation and Dynamics in Nonequilibrium Systems, Cambridge University Press, Cambridge, 2009.

[15] P.G. Ciarlet, The Finite Element Method for Elliptic Problems, North-Holland, Amsterdam, 1978.

[16] W. Cunningham, P. Wangersky, Time lag in prey-predator population models, Ecology 38 (1957) 136139. 
[17] M. Dupraz, S. Filippi, A. Gizzi, A. Quarteroni, R. Ruiz-Baier, Finite element and finite volume-element simulation of pseudo-ECGs and cardiac alternans, Math. Methods Appl. Sci., 38(6) (2015) 1046-1058.

[18] R.E. Ewing, R.D. Lazarov, Y. Lin, Finite volume element approximations of nonlocal reactive flows in porous media, Numer. Methods Partial Differ. Equ. 16 (2000) 285-311.

[19] H.I. Freedman, V.S. Hari Rao, The trade-off between mutual interference and time lags in predator-prey system, Bull. Math. Biol. 45 (1983) 991-1004.

[20] M.R. Garvie, Finite-difference schemes for reaction-diffusion equations modeling predator-prey interactions in MATLAB, Bull. Math. Biol. 69 (2007) 931-956.

[21] M.R. Garvie, C. Trenchea, A three level finite element approximation of a pattern formation model in developmental biology, Numer. Math. 127 (2014) 397-422.

[22] K. Gopalsamy, Pursuit evasion wave trains in prey-predator systems with diffusionally coupled delays, Bull. Math. Biol. 42 (1980) 871-887.

[23] K. Gopalsamy, Stability and Oscillation in Delay Differential Equations of Population Dynamics. Kluwer, Dordrecht, 1992.

[24] S.A. Gourley, J. So, J. Wu, Nonlocality of reaction-diffusion equations induced by delay: biological modeling and nonlinear dynamics, J. Math. Sci. (N. Y.) 124 (2004) 5119-5153.

[25] S.A. Gourley, R. Liu, J. Wu, Spatiotemporal patterns of disease spread: interaction of physiological structure, spatial movements, disease pogression and human intervention. In P. Magal and S. Ruan (Eds.), Structured Population Models in Biology and Epidemiology, Springer-Verlag, Berlin, 2008, pp. 165-208.

[26] E. Hairer, G. Wanner, Solving Ordinary Differential Equations II, Springer-Verlag, New York, 2002.

[27] J.K. Hale, H. Koçak, Dynamics and Bifurcations, Springer-Verlag, New York, 1991.

[28] B. Hassard, D. Kazarino, Y. Wan, Theory and Applications of Hopf Bifurcation, Cambridge University Press, Cambridge, 1981.

[29] C. Huang, Delay-dependent stability of high order Runge-Kutta methods, Numer. Math. 111 (2009) $377-387$.

[30] C. Huang, S. Vandewalle, Unconditionally stable difference methods for delay partial differential equations, Numer. Math. 122 (2012) 579-601.

[31] S. Jana, M. Chakraborty, K. Chakraborty, T.K. Kar, Global stability and bifurcation of time delayed prey-predator system incorporating prey refuge, Math. Comput. Simul. 85 (2012) 57-77.

[32] T. Koto, Stability of IMEX Runge-Kutta methods for delay differential equations, J. Comput. Appl. Math. 211 (2008) 201-212.

[33] Y. Kuang, Delay Differential Equations with Applications in Population Dynamics, Academic Press, New York, 1993.

[34] J. Li, Z. Chen, Y. He, A stabilized multi-level method for non-singular finite volume solutions of the stationary 3D Navier-Stokes equations, Numer. Math. 122 (2012) 279-304.

[35] Z. Lin, R. Ruiz-Baier, C. Tian, Finite volume element approximation of an inhomogeneous Brusselator model with cross-diffusion, J. Comput. Phys. 256 (2014) 806-823.

[36] H. Malchow, S.V. Petrovskii, E. Venturino, Spatiotemporal Patterns in Ecology and Epidemiology: Theory, Models, Simulations, Chapman \& Hall / CRC Press, 2008.

[37] R.M. May, Stability and Complexity in Model Ecosystems, Princeton University Press, Princeton, 1973.

[38] M.A. McKibben, Discovering Evolution Equations with Applications. Volume 1-Deterministic Equations, CRC Press, Boca Raton, 2011. 
[39] A.B. Medvinsky, S.V. Petrovskii, I.A. Tikhonova, H. Malchow, B.-L. Li, Spatiotemporal complexity of plankton and fish dynamics, SIAM Rev. 44 (2002) 311-370.

[40] J.D. Murray, Spatial structures in predator-prey communities-A nonlinear time delay diffusional model, Math. Biosci. 30 (1976) 73-85.

[41] J.D. Murray, Mathematical Biology I: An Introduction. Third Edition. Springer, New York, 2002.

[42] J.D. Murray, Mathematical Biology II: Spatial Models and Biomedical Applications. Third Edition. Springer, New York, 2003.

[43] S. Nababan, K.L. Teo, Existence and uniqueness of weak solutions of the Cauchy problem for parabolic delay-differential equations, Bull. Australian Math. Soc. 21 (1980) 65-80.

[44] C.V. Pao, Nonlinear Parabolic and Elliptic Equations, Plenum Press, New York, 1992.

[45] S. Phongthanapanich, P. Dechaumphai, Finite volume element method for analysis of unsteady reactiondiffusion problems, Acta Mech. Sin. 25 (2009) 481-489.

[46] A. Quarteroni, R. Ruiz-Baier, Analysis of a finite volume element method for the Stokes problem, Numer. Math. 118 (2011) 737-764.

[47] L.A.D. Rodrigues, D.C. Mistro, S. Petrovskii, Pattern formation, long-term transients, and the TuringHopf bifurcation in a space- and time-discrete predator-prey system, Bull. Math. Biol. 73 (2011) 18121840.

[48] S. Ruan, On nonlinear dynamics of predator-prey models with discrete delay, Math. Model. Nat. Phenom. 4 (2009) 140-188.

[49] S.G. Ruan, J.J. Wei, On the zero of some transcendental functions with applications to stability of delay differential equations with two delays, Dyn. Contin. Discrete Impuls. Sys. Ser. A Math. Anal. 10 (2003) 863-874.

[50] S. Sen, P. Ghosh, S.S. Riaz, D.S. Ray, Time-delay-induced instabilities in reaction-diffusion systems, Phys. Rev. E 80 (2009) paper 046212.

[51] F. Shakeri, M. Dehghan, The finite volume spectral element method to solve Turing models in the biological pattern formation, J. Comput. Math. Appl. 62(12) (2011) 4322-4336.

[52] H. Smith, An Introduction to Delay Differential Equations with Applications in the Life Sciences, Springer-Verlag, New York, 2011.

[53] G.-Q. Sun, Z. Jin, M. Haque, B.-L. Li, Spatial patterns of a predator-prey model with cross diffusion, Nonlinear Dyn. 69 (2012) 1631-1638.

[54] C. Tian, Delay-driven spatial patterns in a plankton allelopathic system, Chaos 22 (2012) paper 013129.

[55] V. Volterra, Variazioni e fluttuazioni del numero d'individui in specie animali conviventi, Mem. Acad. Lincei 2 (1926) 31-116.

[56] V. Volterra, Leçons sur la Théorie Mathématique de la Lutte pour la Vie, Gauthier-Villars, Paris, 1931.

[57] W. Wang, Epidemic models with time delays, in Z. Ma, Y. Zhou, J. Wu (eds.), Modeling and Dynamics of Infectious Diseases, Higher Education Press, Beijing, 2009, 289-314.

[58] W. Wang, L.S. Chen, A predator-prey system with stage-structure for predator, J. Comput. Appl. Math. 33 (1997) 83-101.

[59] Y.-M. Wang, C.V. Pao, Time-delayed finite difference reaction-diffusion systems with nonquasimonotone functions, Numer. Math. 103 (2006) 485-513.

[60] A. Xiao, G. Zhang, J. Zhou, Implicit-explicit time discretization coupled with finite element methods for delayed predator-prey competition reaction-diffusion system, Comput. Math. Appl. 71(10) (2016) $2106-2123$ 
[61] J.-F. Zhang, W.-T. Li, X.-P. Yan, Hopf bifurcation and Turing instability in spatial homogeneous and inhomogeneous predator-prey models, Appl. Math. Comput. 218 (2011) 1883-1893.

[62] T. Zhao, Y. Kuang, H.L. Smith, Global existence of periodic solutions in a class of delayed Gause-type predator-prey systems, Nonlinear Anal. 28 (1997) 1373-1390. 